\title{
Impurity Effects of Aerogel in Superfluid ${ }^{3} \mathrm{He}$
}

\author{
W.P. Halperin, H. Choi, J.P. Davis, and J. Pollanen \\ Department of Physics and Astronomy, \\ Northwestern University, Evanston, IL 60208, USA
}

(Dated: Version July 15, 2021)

\begin{abstract}
The discovery of superfluid ${ }^{3} \mathrm{He}$ in high porosity silica aerogels, and subsequent experimental and theoretical work, have led to a better general understanding of quasiparticle scattering from impurities in unconventional pairing systems. It is immensely helpful for understanding impurity effects in the case of superfluid ${ }^{3} \mathrm{He}$ that the structure of its order parameter is well-established. An overview of impurity effects is presented with emphasis on those experiments which have a quantitative interpretation in terms of theoretical models for homogeneous and inhomogeneous scattering. The latter can account successfully for most experimental results.

PACS numbers: $67.30 . \mathrm{hm}, 67.30 . \mathrm{ht}, 43.35 .+\mathrm{d}, 81.05 . \mathrm{Rm}$
\end{abstract}

\section{INTRODUCTION}

Since its discovery ${ }^{1}$ in 1971 , superfluid ${ }^{3}$ He has been influential for investigations of quantum condensed systems including exotic and unconventional superconductivity, quantum gases,$[2] 3$ neutron stars,$\frac{4]}{, 2}$ and systems with complex spontaneous symmetry breaking, as for example in quark matter ${ }^{5}$ The impact of superfluid ${ }^{3} \mathrm{He}$ research in condensed matter physics has been most closely related to unconventional superconducting pairing condensations, high temperature superconductivity and certain heavy fermion superconductors ${ }^{2]}$ In these cases the effects of quasiparticle scattering, either from chemical impurities or material defects, can be significant ${ }^{6}$ and yet they can be difficult to interpret. For these exotic superconductors, one of the difficulties is that the order parameter of the pure system may not be established. It is helpful for developing basic ideas to compare with ${ }^{3} \mathrm{He}$ where the order parameter structure is very wellknown and where there are high resolution experimental techniques to probe both spin and orbital dependences of the condensed state. Consequently, ${ }^{3} \mathrm{He}$ is an excellent forum for investigating impurity effects; however, it was not known until recently how to systematically introduce such impurities into ${ }^{3} \mathrm{He}$, the purest of any known material.

About a dozen years ago groups at Cornell University and Northwestern University investigated samples of 98\% porosity silica aerogel samples provided by Moses Chan from Pennsylvania State University, to see what might be the behavior at low temperatures of ${ }^{3} \mathrm{He}$ imbibed into these highly porous structures. Both groups reported evidence for sharp onsets of superfluidity albeit at transition temperatures significantly reduced compared with the pure superfluid, as shown in the phase diagram of the transition temperature, $T_{c a}$, versus pressure in Fig. 1. A flood of questions then arose: What was the new pressure-temperature phase diagram? Is it reproducible from one aerogel sample to the next? To what extent is the order parameter suppressed and does it track the reduction in the transition temperature? What are the equilibrium phases? Are there new physical phe- nomena different from the pure system? Concerning the last question, there is currently substantial interest in a metastable equal-spin-pairing state, an $A$-like phase, that appears on cooling through the normal-to-superfluid transition. It was suggested by Vicente et al $\frac{10}{10}$ that the origin of this phase might be associated with anisotropic scattering. Subsequently, some theoretical work ${ }^{11}$ and a number of experiments $\frac{12|13| 14|15| 16 \mid 17}{\text { have investigated }}$ ${ }^{3} \mathrm{He}$ in aerogels having global anisotropy, suggesting new order parameter structures and perhaps new kinds of superfluid phases. These questions and others have been the subject of intense activity.

In this article we concentrate mainly on a number of experimental results which have been interpreted quantitatively in terms of theoretical models for impurity scattering. As such our review is not a comprehensive treatment of superfluid ${ }^{3} \mathrm{He}$ in aerogel, although it is complementary

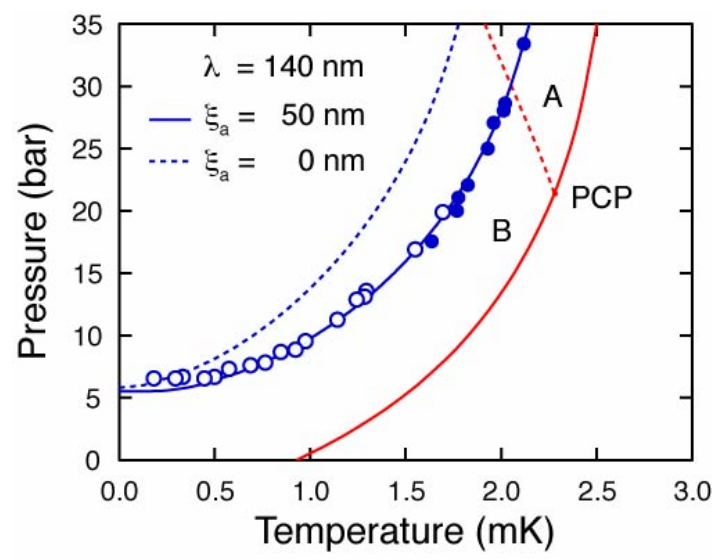

FIG. 1: Phase diagram for superfluid ${ }^{3} \mathrm{He}$ in two different samples of $98 \%$ aerogel. The known superfluid phases of pure ${ }^{3} \mathrm{He}$ are labeled $\mathrm{A}$ and $\mathrm{B}$ meeting with the normal phase at a polycritical point, PCP. The open circles are measurements from the Cornell group $\sqrt{19}$ and the closed circles from the Northwestern group $20 \mid 21$ both for nominally $98 \%$ porous aerogels made by Norbert Mulders at the University of Delaware. The blue curves are from theoretical models discussed in the text. 

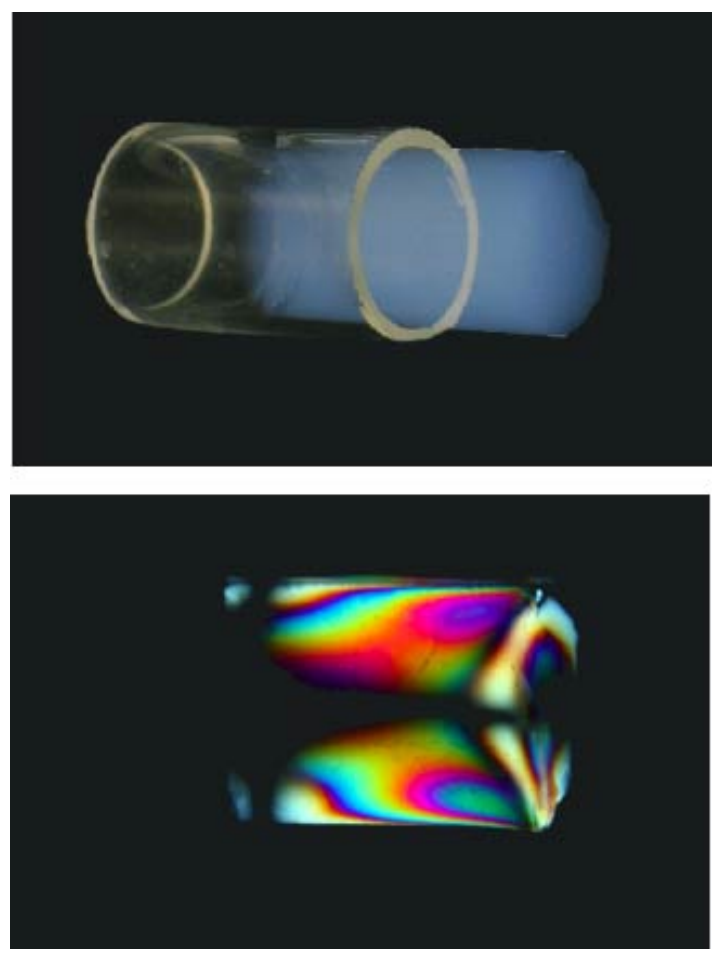

FIG. 2: High porosity silica aerogel made at Northwestern. The upper image shows a sample after supercritical drying partially removed from the glass tube in which it was formed. The lower image is from exposure to white light passing through crossed polarizers, an example of an inhomogeneous aerogel with both density variations and anisotropy that can result from abnormally high concentrations of catalyst used during synthesis. Both samples have $98 \%$ porosity. In contrast, a homogeneous aerogel would not transmit any light and the image would be black.

to an introductory review by Halperin and Sauls ${ }^{18}$ We outline the principal features of superfluid ${ }^{3} \mathrm{He}$ in aerogel; we introduce the GL-theory for a $p$-wave superfluid and its adaption to theoretical models for impurity scattering; and we discuss the experimental results in this context. Lastly, we discuss the metastable anisotropic phase, and some of the recent work on anisotropic aerogels that attempt to uncover the origin of this fascinating phenomenon of metastability between different $p$-wave superfluid states.

\section{HIGH POROSITY SILICA AEROGEL}

Highly porous silica aerogel is itself a subject of widespread interest to diverse applications including the collection of cosmic interstellar particles, thermal insulation, catalysis, particle detectors, radiation absorbers, liquid crystal phase transitions, and applications to quantum fluids and solids, where the present topic is but one

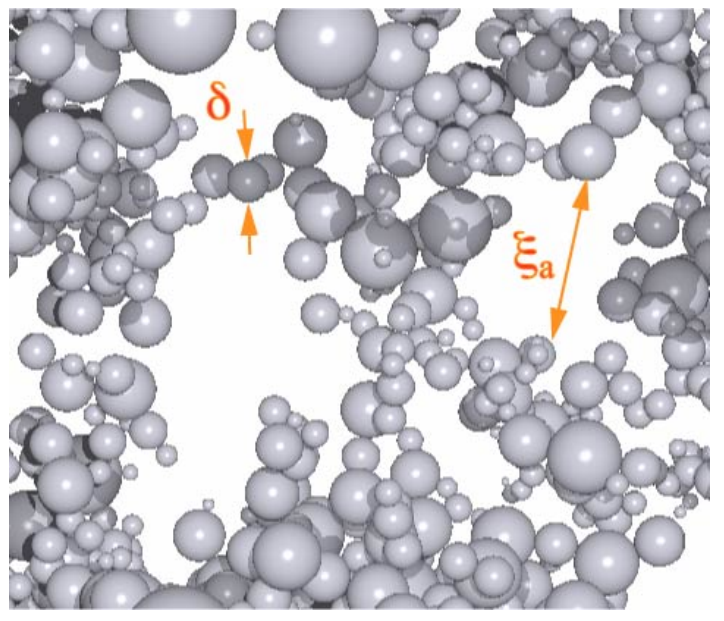

FIG. 3: Perspective view of a DLCA calculation of a $98 \%$ aerogel. The principal length scales can be seen including the $\mathrm{SiO}_{2}$ particles $\approx 3 \mathrm{~nm}$ in diameter and their correlation length $\xi_{a} \approx 40 \mathrm{~nm}$. The transport mean free path is much longer $\left(\lambda>>\xi_{a}\right)$ given the open nature of the structure, $\lambda \approx 180 \mathrm{~nm}$. This simulation was made by Tom Lippman at Northwestern University. Simulations have been performed by Porto and Parpia ${ }^{23}$ and Haard et $a l !^{24 \mid 25}$

example. See Pollanen et al ${ }^{[22}$ and references therein concerning these applications. In Fig. 2 we show a typical sample of $98 \%$ porous silica aerogel grown at Northwestern University partly extracted from the glass tube in which it was produced. Under some synthesis conditions, including high concentrations of base catalyst, the aerogels will exhibit anisotropy that can be easily visualized with white light and crossed polarizers as shown in the colorful picture in the second panel of Fig. 2. However, the synthesis can be controlled such that the anisotropy is either globally axial, or completely avoided altogether. ${ }^{222}$ In the latter case, an image with polarized light of a homogeneous and isotropic aerogel sample is black for any angle of presentation with respect to the polarizers. In the case of homogeneous axial anisotropy the image is black only for the anisotropy axis (optical axis) parallel to either of the polarizers. It is important for applications to ${ }^{3} \mathrm{He}$ that the structure of the aerogel be well-characterized using optical birefringence methods in combination with small angle x-ray measurements (SAXS) as discussed by Pollanen et al ${ }^{[22}$ We have grown isotropic silica aerogels by the one-step method in the range of 95 to $99 \%$ porosity and $98 \%$ porosity using the two-step method; in both cases we use a technique of rapid supercritical extraction ${ }^{26}$ during the drying stage.

Additionally, numerical simulations can be very helpful for understanding aerogel. They are generally based on diffusion limited cluster aggregation (DLCA) algo-

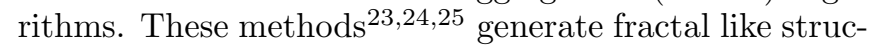
tures such as shown in Fig. 3, that permit calculation of the x-ray scattering crossection and the transport (ballistic) mean free path, $\lambda$. This may be the best way to 


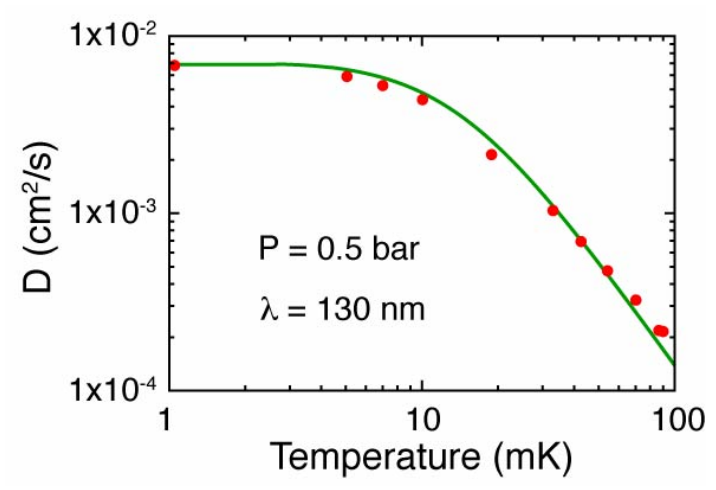

FIG. 4: Diffusion coefficient for normal ${ }^{3} \mathrm{He}$ determined from NMR by the Grenoble group ${ }^{29 \mid 30}$ The leveling off of diffusion with decreasing temperature indicates the transition from inelastic to elastic scattering from which $\lambda=130 \mathrm{~nm}$ was obtained from consideration of elastic scattering in the normal Fermi liquid.

correlate measured transport coefficients in quantum fluids with microscopic characterization using SAXS. The simulation in Fig. 3 is a perspective view of a calculation performed by Tom Lippman at Northwestern University using DLCA. The principal length scales of the structure are indicated: the $\mathrm{SiO}_{2}$ particle size of $\delta \approx 3 \mathrm{~nm}$, and the particle-particle correlation length $\xi_{a} \approx 40 \mathrm{~nm}$.

Ideally $\lambda$ should be determined directly from transport experiments in the normal fluid: acoustic attenuation, 27 thermal conductivity, $\stackrel{28}{,}$ or diffusion measurements. $\stackrel{2930}{2130}$ The analysis of diffusion measurements performed by the Grenoble group ${ }^{30}$ are shown in Fig. 4 for a $98 \%$ aerogel giving, $\lambda=130 \mathrm{~nm}$. It is noteworthy that the transport mean free path $\lambda$ determined in the normal state from diffusion, matches well with the corresponding parameter obtained from analysis of superfluid ${ }^{3} \mathrm{He}$ properties using the inhomogeneous scattering theory.

\section{IMPURITY SCATTERING FROM SILCA AEROGEL}

The effect of magnetic impurity scattering on the superconducting transition of an $s$-wave superconductor was calculated by Abrikosov and Gorkov (AG) ${ }^{31}$ and extended by Tsunetd ${ }^{32}$ and Larkin ${ }^{33}$ to non- $s$-wave pairing, where any form of impurity scattering has the effect of depairing. To adapt this theory to $p$-wave superfluids 34 we define a pair breaking parameter, $x$, to be the ratio of the coherence length at zero temperature of pure ${ }^{3} \mathrm{He}$ to the transport mean free path, $x=\xi_{0} / \lambda$ where $\xi_{0}=\hbar v_{F} /\left(2 \pi k_{B} T_{c}\right)$. According to the AG-theory ${ }^{[31}$ the dependence of $T_{c a} / T_{c}$ on $x$, is given in Fig. 5 , where $T_{c}$ is the transition temperature in the absence of elastic scattering. In the clean limit there is a linear relationship. For large scattering rates there is a critical value of $x$ beyond which the superconducting state is not sta-

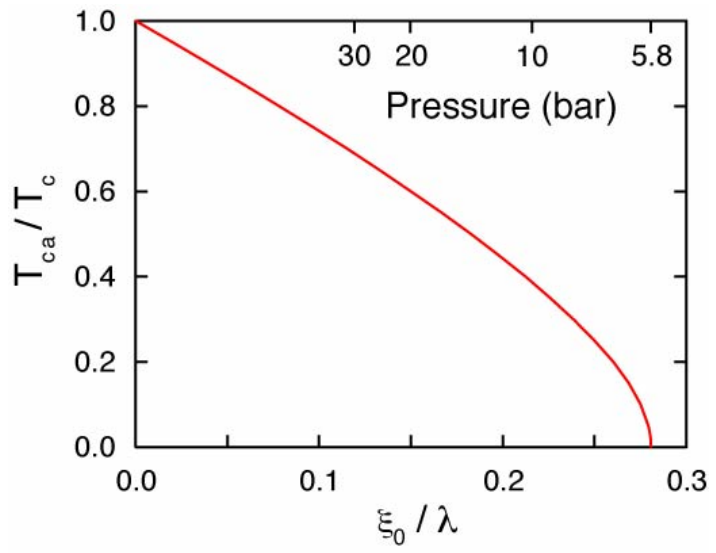

FIG. 5: Reduction of the transition temperature by impurity scattering as a function of the pair breaking parameter, $x=\xi_{0} / \lambda$, according to Abrikosov and Gorkov! 31 It can be easily seen that this behavior, to first order, accounts for the phase diagram in Fig. 1 since a clockwise rotation of the above figure by $90^{\circ}$ maps onto the phase diagram, as suggested by the upper-scale for the pressure in this figure with $\lambda=140 \mathrm{~nm}$. The depairing parameter depends on the pressure through the pressure dependence of the coherence length, $\xi_{0}$. Critical depairing is reached near $P=6$ bar where $x=0.28$.

ble. These features are qualitatively manifest in the observed phase diagram for superfluid ${ }^{3} \mathrm{He}$ in aerogel shown in Fig. 1. Here $\lambda$ is fixed by the silica structure while $\xi_{0}$ varies between $16 \mathrm{~nm}$, at high pressure, to $77 \mathrm{~nm}$ at zero pressure. Matsumoto et al. ${ }^{19}$ discovered a critical pressure for superfluidity at $P \approx 6$ bar in a $98 \%$ aerogel which corresponds to the critical value for the depairing parameter in Fig. 5, predicted by Abrikosov and Gorkov! 31

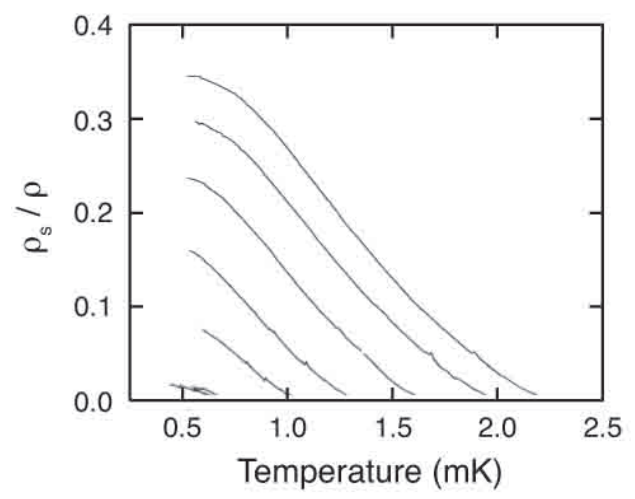

FIG. 6: Superfluid density and transition temperature for superfluidity from torsional oscillator measurements from the Cornell University group of Porto and Parpia! The pressures shown for these curves are, from lowest to highest: $3.45 .0,7.0,10,15$, and 25 bar. This sample showed less suppression of $T_{c a}$ than that reported later by Matsumoto et al ${ }^{[19]}$ (Fig. 1.) 


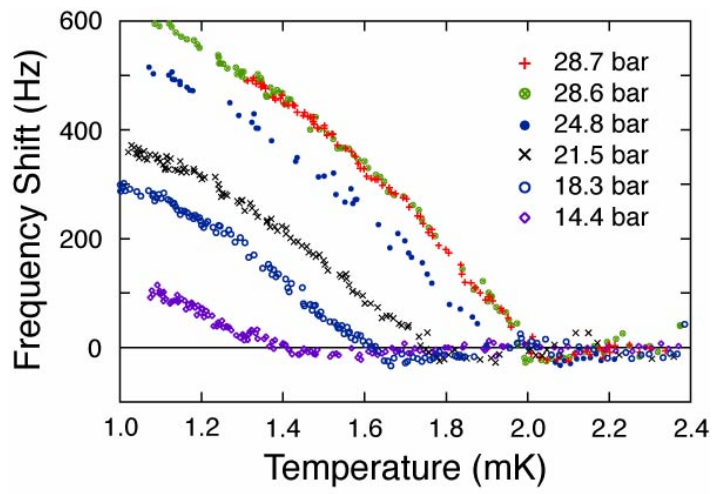

FIG. 7: NMR frequency shifts measured by the Northwestern group ${ }^{8|9| 25}$ for superfluid ${ }^{3} \mathrm{He}$ in a $98 \%$ aerogel. There is a clear indication of the transition temperature. The magnitude of the frequency shifts at each pressure, corrected for surface solid ${ }^{3} \mathrm{He}$, is reduced by a simple scale factor relative to the pure $A$-phase as reported by Sprague et al. ${ }^{[]}$and shown in Fig. 8. These authors identified an equal-spin-pairing state, like the $A$-phase.

\section{INDICATORS OF $\mathbf{T}_{c}$}

The first indication of a transition temperature for superfluidity of ${ }^{3} \mathrm{He}$ in a $98 \%$ aerogel was found by Porto and Parpia ${ }^{7}$ from the period shift measurements of a torsional oscillator that they interpreted as an unlocking of the superfluid component. They obtained the superfluid density as shown in Fig. 6. Shortly thereafter, Sprague et al. $\frac{89}{6}$ found frequency shifts of the NMR spectrum, Fig. 7, which displayed a sudden onset at a given temperature and were similarily interpreted. Transition temperatures obtained later by these two groups are intercompared in the phase diagram in Fig. 1 indicating the same degree of suppression. It was clear from these first experiments that the transition temperature is systematically reduced compared to that of the pure superfluid, and this effect is more pronounced at lower pressures. This is expected from AG-theory. But it was also clear that the low temperature limiting value for the superfluid density, particularly at low pressure, was not $100 \%$. Similarily the magnitude of the NMR frequency shifts was substantially reduced from values expected for pure ${ }^{3} \mathrm{He}$. In both cases it appears that the order parameter is suppressed, but more than expected from scaling of the transition temperature alone. From the AG-theory, adapted to $p$-wave superfluidity ${ }^{34}$ with unitary scattering, the suppression of these two basic superfluid characteristics should be the same. In Fig. 8 a comparison is made to demonstrate this point. It can be seen that the transition temperature for the superfluid in aerogel, relative to the pure superfluid, is reduced, but systematically less so as compared to the amplitude of the order parameter, $\Delta(T)$, in the Ginzburg-Landau limit. The square of the order parameter amplitude near $T_{c}$ is

$$
\Delta^{2}(T)=\left(\pi k_{B} T_{c}\right)^{2} \frac{2}{3} \frac{\Delta C}{C}\left(\frac{T_{c}}{T}-1\right),
$$

which can be directly determined from the specific heat jump, $\Delta C / C$, such as measured by Choi et al $\stackrel{35}{\text { Addi- }}$ tionally, measurements of the NMR frequency shift, $\Delta \omega$, where $\Delta^{2} \propto \Delta \omega$, can be taken from Sprague et al ${ }^{[8]}$ to infer suppression of the order parameter. The two experiments, shown in Fig. 8, are in excellent agreement, as will be discussed in subsequent sections. However the different suppression factors for the transition temperature and the order parameter indicate that a strict application of the AG-theory, adapted to $p$-wave pairing, the socalled homogeneous isotropic scattering model (HISM), does not suffice.

\section{GL-THEORY AND EXPERIMENT}

According to Ginzburg-Landau theory, just below the temperature for a second order thermodynamic transition, the free energy can be represented phenomenologically as an expansion in terms of the order parameter. In the case of superfluid ${ }^{3} \mathrm{He}$, the order parameter, $A$, is a complex, second-rank tensor and the free energy can be expressed $\sqrt[36 / 37 / 38]{ }$ in terms of its invariants. Neglecting the dipole energy, this has the form,

$$
\begin{aligned}
F= & -\alpha \operatorname{Tr}\left(A A^{\dagger}\right)+g_{z} H_{\mu}\left(A A^{\dagger}\right)_{\mu \nu} H_{\nu}+\beta_{1}\left|\operatorname{Tr}\left(A A^{T}\right)\right|^{2} \\
& +\beta_{2}\left[\operatorname{Tr}\left(A A^{\dagger}\right)\right]^{2}+\beta_{3} \operatorname{Tr}\left(A A^{T}\left(A A^{T}\right)^{*}\right) \\
& +\beta_{4} \operatorname{Tr}\left(\left(A A^{\dagger}\right)^{2}\right)+\beta_{5} \operatorname{Tr}\left(A A^{\dagger}\left(A A^{\dagger}\right)^{*}\right)
\end{aligned}
$$

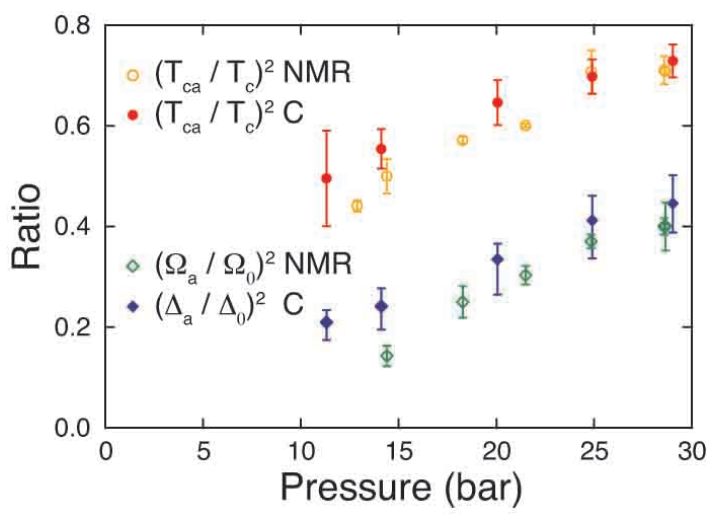

FIG. 8: Suppression of the transition temperature and the amplitude of the order parameter as a function of pressure. The amplitude of the order parameter is determined from the NMR frequency shifts and independently from the specific heat jump measurements of Choi et $a l{ }^{[35}$ According to the homogeneous isotropic scattering model, with unitary scattering, ${ }^{[34}$ the suppression factor for the transition temperature and the order parameter amplitude should be the same, in contrast to the experiment. Note that the $98 \%$ aerogel samples for specific heat and NMR experiments are slightly different from one another. 
that we have taken from Thuneberg ${ }^{37}$ and the discussion by Choi et al! ${ }^{[39]}$ The magnetic field components are $H_{\nu}$, and $A^{\dagger}$ and $A^{T}$ are the Hermitian conjugate and transpose of $A$. There are five fourth-order terms for which the coefficients, $\beta_{i}$, determine the stable superfluid states. In the weak-coupling limit,

$$
\begin{gathered}
\alpha=\frac{N(0)}{3}\left(\frac{T}{T_{c}}-1\right) \\
\frac{\beta_{i}}{\beta_{0}}=(-1,2,2,2,-2), i=1, \ldots, 5 \\
\beta_{0}=\frac{7 \zeta(3)}{120 \pi^{2}} \frac{N(0)}{\left(k_{B} T_{c}\right)^{2}} \\
g_{z}=\frac{7 \zeta(3)}{48 \pi^{2}} N(0)\left(\frac{\gamma_{0} \hbar}{\left(1+F_{0}^{a}\right) k_{B} T_{c}}\right)^{2}
\end{gathered}
$$

where the normal density of states at the Fermi energy is $N(0)$, the gyromagnetic ratio for ${ }^{3} \mathrm{He}$ is $\gamma_{0}, k_{B}$ is the Boltzmann constant, $F_{0}^{a}$ is a Fermi liquid parameter determined from magnetization measurements 38 and $\zeta(x)$ is the Riemann zeta function. For the weak-coupling superfluid the isotropic state, called the $B$-phase, is always the most stable of all the $p$-wave states. The existence of the axial state at high pressure, known as the $A$-phase, is a result of strong-coupling effects which are, to leading order, proportional to $T_{c} / T_{F}$, 40

The coefficient for the field coupling term of the order parameter, $g_{z}$, is determined by measuring the slope of the magnetization of ${ }^{3} \mathrm{He}-B$ in the limit approaching $T_{c}$. The measurements to date for pure ${ }^{3} \mathrm{He}$ are consistent with $g_{z}$ equal to its weak-coupling value even at high pressure.25] This coefficient has the form,

$$
g_{z}=g_{z}^{\mathrm{w} c} \frac{\frac{d m}{d t}}{\frac{d m}{d t}{ }^{\mathrm{w} c}} \frac{\beta_{B}}{\beta_{B}^{\mathrm{w} c}},
$$

where $m=M_{B} / M_{N}, M_{N}$ is the normal state magnetization, and $\beta_{B}$ is defined below. The superscript wc, which we use here and in the following, indicates the weak-coupling limit.

It was shown by Greaves 41 that the rather small NMR $g$-shift ${ }^{25|39| 42}$ of the transverse NMR frequency in ${ }^{3} \mathrm{He}-B$ in the dipole-locked limit can be used to determine $\beta_{345}$ in combination with $\hat{g}_{z}=g_{z} / g_{z}^{\mathrm{w} c}$.

$$
\frac{\beta_{345}}{\hat{g}_{z}}=\beta_{345}^{\mathrm{w} c} \frac{1}{\left(1+F_{0}^{a}\right)^{2}}\left(\frac{C_{N}}{\Delta C_{B}}\right) \frac{\nu_{B}^{2}}{1-t}\left(\frac{\hbar}{2 \pi k_{B} T_{c}}\right)^{2} \frac{1}{g} .
$$

where $\nu_{B}$ is the longitudinal resonance frequency in the $B$-phase measured by Rand et al., ${ }^{4344}$ and $t=T / T_{c}$. We use the standard notation, $\beta_{i j}=\beta_{i}+\beta_{j}$. The $g$-shift measurements are summarized by Haard 25 and by Choi et al!

The specific heat, $\stackrel{45}{4} C_{N}$ and its jump $\Delta C_{A}$ and $\Delta C_{B}$ at $T_{c}$ for the $A$ and $B$-phases, are related to $\beta_{A}$ and $\beta_{B}$ through,

$$
\begin{gathered}
\Delta C_{A}=\frac{\alpha^{\prime 2}}{2 \beta_{A}}, \beta_{A} \equiv \beta_{245} \\
\Delta C_{B}=\frac{\alpha^{\prime 2}}{2 \beta_{B}}, \beta_{B} \equiv \beta_{12}+\frac{1}{3} \beta_{345}
\end{gathered}
$$

where $\alpha^{\prime} \equiv d \alpha / d T$. The magnetic suppression ${ }^{46}$ of $T_{A B}$, the transition from $A$ to $B$-phases is, $g(\beta)$,

$$
g(\beta)=-\frac{\sqrt{1+\left(\beta_{B} / \beta_{A}-1\right)\left(1+\frac{2}{1-\beta_{12} / \beta_{B}}\right)}+1}{6\left(\beta_{B} / \beta_{A}-1\right)} .
$$

Here $g(\beta)$ is defined by,

$$
1-\frac{T_{A B}}{T_{c}} \equiv g(\beta)\left(\frac{B}{B_{0}}\right)^{2}+O\left(\frac{B}{B_{0}}\right)^{4}
$$

where $B_{0}^{2}=N(0) / 6 g_{z}$. Then finally $\beta_{5}$ can be determined by measuring the asymmetry ratid $\frac{47}{4}$ of the $A_{1}-A_{2}$ splitting in a high magnetic field, $r$,

$$
r \equiv \frac{T_{A 1}-T_{c}}{T_{c}-T_{A 2}}=-\frac{\beta_{5}}{\beta_{245}}
$$

These strong-coupling effects on the $\beta$-parameters are determined by experiment. Recently a summary of these parameters for pure ${ }^{3} \mathrm{He}$ was given by Choi et al ${ }^{[39]}$ as well as their analysis for $98 \%$ porosity aerogel. In fact there are only four independent experiments that relate to the $\beta$-parameters in pure ${ }^{3} \mathrm{He}$. Choi et al. ${ }^{39}$ have gone one step further taking advantage of theoretical calculations of Sauls and Serene $\frac{48}{4}$ to propose one additional constraint based on the calculated pressure dependence of these parameters. They obtain all five $\beta$-parameters with reasonable confidence and correspondingly extend these to $98 \%$ aerogel. Their results are reproduced in Table 1 for a particular set of scattering parameters. An important aspect of superfluid ${ }^{3} \mathrm{He}$ in aerogel is that suppression of the transition temperature by elastic scattering reduces strong-coupling effects as compared to pure ${ }^{3} \mathrm{He}$.

\section{HOMOGENEOUS AND INHOMOGENEOUS SCATTERING MODELS}

The experiments for ${ }^{3} \mathrm{He}$ in aerogel are directly related to the corresponding measurements in pure ${ }^{3} \mathrm{He}$ thereby determining the effect of elastic scattering on specific combinations of $\beta$-parameters at various pressures. From the scattering models it is then possible to calculate the model parameters, notably the transport mean free path, $\lambda$, and the impurity particle-particle correlation length $\xi_{a}$. In the following we briefly review these scattering models and then discuss experiments performed in $98 \%$ aerogel and their analysis in terms of these parameters. Despite possible variations between aerogel samples of the same nominal porosity (see Fig. 8 as an example) we find that the values $\lambda=150 \mathrm{~nm}$ and $\xi_{a}=40 \mathrm{~nm}$ fairly represent all of the experiments for $98 \%$ aerogels and will be discussed in detail in the following. 


\section{A. Homogeneous Isotropic Scattering Model}

A number of theoretical models have been proposed to account for depairing of superfluid ${ }^{3} \mathrm{He}$ in aerogel. The first results from Thuneberg et al. $\frac{49}{4}$ and their subsequent publication ${ }^{34}$ provided a detailed framework that extended the Ginzburg-Landau theory for impurities in superconductors 31 to $p$-wave superfluidity. The two important assumptions are that i) the impurity scattering is homogeneous, and that ii) scattering is isotropic. Consequently, this model is called the homogeneous isotropic scattering model (HISM). The HISM of Thuneberg et al! $!^{34}$ modifies the $\beta$-parameters to accomodate depairing expressed in terms of the AGdepairing parameter $x=\xi_{0} / \lambda$. Theoretical interpretation of experiments are mostly based on this model or its variations. $30|39| 50|51| 52|53| 54|55| 56|57|$ The modified $\beta$ parameters, $\beta_{i}^{a}$ for the case of superfluid ${ }^{3} \mathrm{He}$ in aerogel can be expressed as,

$$
\begin{gathered}
\left(\begin{array}{c}
\beta_{1}^{a} \\
\beta_{2}^{a} \\
\beta_{3}^{a} \\
\beta_{4}^{a} \\
\beta_{5}^{a}
\end{array}\right)=\beta_{0}^{a}\left(\begin{array}{c}
-1 \\
2 \\
2 \\
2 \\
-2
\end{array}\right)+b\left(\begin{array}{c}
0 \\
1 \\
0 \\
1 \\
-1
\end{array}\right)+\left(\begin{array}{c}
\Delta \beta_{1}^{s c, a} \\
\Delta \beta_{2}^{s c, a} \\
\Delta \beta_{3}^{s c, a} \\
\Delta \beta_{4}^{s c, a} \\
\Delta \beta_{5}^{s c, a}
\end{array}\right) \\
\beta_{0}^{a}=\frac{N(0)}{30\left(\pi k_{B} T_{c}\right)^{2}} \sum_{n=1} \frac{1}{(2 n-1+x)^{3}} \\
b=\frac{N(0)}{9\left(\pi k_{B} T_{c}\right)^{2}}\left(\sin ^{2} \delta_{0}-\frac{1}{2}\right) \sum_{n=1} \frac{x}{(2 n-1+x)^{4}}
\end{gathered}
$$

The effects of scattering within the weak-coupling approximation are included in $\beta_{0}^{a}$ and $b ; \delta_{0}$ is the $s$-wave scattering phase shift. The strong-coupling corrections to the impurity-superfluid $\beta$-parameters, $\Delta \beta^{s c, a}$, must be determined from experiment. We take the point of view that strong-coupling is manifest in the impurity system in the same way as for the pure superfluid with the exception that these contributions are rescaled by the factor $T_{c a} / T_{c}$ allowing for the fact that strong-coupling in leading order is proportional to the transition temperature 40 Then the prescription is straightforward. The strongcoupling contributions to $\Delta \beta^{s c}$ for the pure system are multiplied by the factor $T_{c a} / T_{c}$ and added to the corresponding weak-coupling contributions for the impure system, $\Delta \beta^{w c, a}$, calculated for the model scattering parameters, $\lambda, \xi_{a}$, and $\delta_{0}$, with $T_{c a}$ determined self-consistently from,

$$
\ln \frac{T_{c}}{T_{c, a}}=2 \sum_{n=1}\left(\frac{1}{2 n-1}-\frac{1}{2 n-1+x}\right) .
$$

Values for all of the $\beta_{i}$ 's have been discussed by Choi et $a l{ }^{[39}$ Within the context of the various scattering models one can then estimate stability of various superfluid states and their properties.
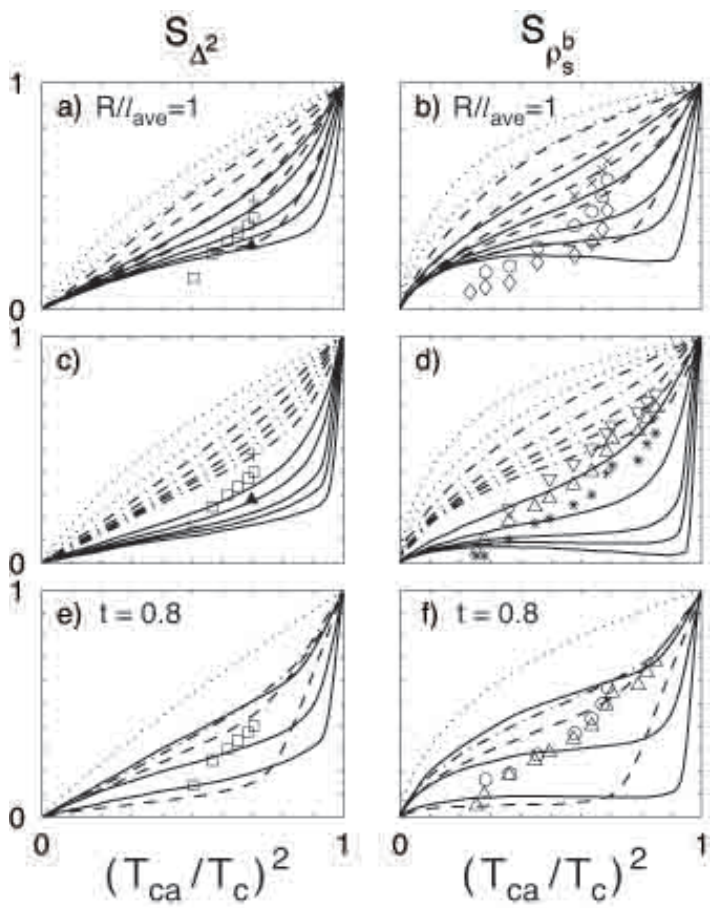

FIG. 9: Suppression factors for the order parameter and the superfluid density calculated by Hänninen and Thuneberg ${ }^{50}$ for various scattering parameters in their theory and at different temperatures. The solid curves are the IISM calculations with temperatures $t=$ $0.2,0.5,0.7,0.8,0.9$ from top to bottom in a) to d). The dot-dashed curves are the HISM results. For a) and b), $R=\lambda$; c) and d), $R=2 \lambda$; and e) and f) top to bottom, $R / \lambda=0.5,1,2$. The data are from NMR, ${ }^{819}$ left side, and torsional oscillator experiments, $\stackrel{23 \mid 59}{2}$ right side. This version of the IISM indicates less suppression of the transition temperature as compared with the order parameter, that there is little distinction between $A$ and $B$-phases, and that the average superfluid density is more sensitive to inhomogeneity than the order parameter amplitude.

\section{B. Inhomogeneous Isotropic Scattering Models}

Several modifications of the HISM have been developed to include inhomogeneous scattering as well as anisotropic scattering! $34 \sqrt[50 / 52]{2}$ The most complete study of inhomogeneous isotropic scattering (IISM) is that of Hänninen and Thuneberg. ${ }^{[50}$ In this approach the ${ }^{3} \mathrm{He}$ is contained in a spherical volume of radius $R$ within which there is a spherically symmetric distribution of scattering centers. The nature of the distribution is defined by parameters in the theory. The radius $R$ is chosen to be close to the average transport mean free path, $\lambda$. The model is numerically intensive but, for suitable parameter values as shown in Fig. 9, can lead to a fair comparison with experiment for both superfluid density and the amplitude of the order parameter. The main feature is that the suppression of the order parameter and the superfluid density is greater than the suppression of the transition temperature for the IISM, in agreement with 
experiment, and greater than the HISM, dashed and dotted lines in Fig. 9. One prediction of the IISM that is inconsistent with experiment is the significant temperature dependence to the suppression factors. The NMR experiments $\$ 58$ find that the measured frequency shifts scale precisely with the values for the pure superfluid over a wide range of temperature, with a temperature independent suppression factor.

Sauls and Sharma ${ }^{52}$ have proposed a simple phenomenological IIS-model that simply redefines the depairing parameter of Abrikosov and Gorkov and is more immediately accessible for data analysis in the context of the Ginzburg-Landau theory. They propose the modified depairing parameter $x=\hat{x} /\left(1+\zeta_{a}^{2} / \hat{x}\right), \zeta_{a}=\xi_{a} / \lambda$, where $\hat{x}=\hbar v_{F} / 2 \pi k_{B} T \lambda$ is the depairing parameter in the HISM, and $\xi_{a}$ is the aerogel particle-particle correlation length. This form preserves the correct limit for homogeneous scattering as $\xi_{a}$ goes to zero and in the opposite limit approaches the form expected for randomly distributed voids of size $\xi_{a}$. The effectiveness of this model can be seen in Fig. 1. The excellent agreement of the theory, solid blue curve (IISM), with the data for transition temperatures is in contrast with the HISM, blue dashed curve. It appears that both IISM theories can successfully correct the difficulty with the HISM noted above and shown in Fig. 8. However, we will follow the approach suggested by Sauls and Sharma owing to its accessibility for data interpretation.

\section{EXPERIMENTAL RESULTS}

\section{A. Identification of the Superfluid State and Phase Diagram}

The first NMR experiments $8|9| 59 \mid 60$ showed that paramagnetic solid ${ }^{3} \mathrm{He}$, adsorbed on the aerogel surface, dominated the temperature dependent magnetization and consequently reduced the NMR frequency shifts substantially by the ratio of the liquid to the total magnetization according to a model for fast exchange. These effects can be allowed for in the measurement as is the case for NMR data in Fig. 7, or they can be eliminated by precoating the surfaces 9 with two or more atomic layers of ${ }^{4} \mathrm{He}$. It seems that preplating with ${ }^{4} \mathrm{He}$ has only slight effect on the overall phase diagram.9

Identification of the symmetry of the observed phases was not possible from measurements of the superfluid density with the torsional oscillator technique ${ }^{7]}$ On the other hand early experiments on NMR frequency shifts and spectra were interpreted either as an equal-spinpairing (ESP) state, ${ }^{8}$ like the $A$-phase with a uniform texture (dipole-locked); or the $B$-phase with an inhomogeneous texture ${ }^{59}$ determined by sample shape (dipoleunlocked). It seems likely that these very different results and their interpretation are a consequence of metastability of the $A$-like phase and differences between the two samples.
A clear signature of an ESP-state is its temperature independent magnetization found from the integral of the NMR spectrum. In contrast, the $B$-phase magnetization is temperature dependent and a transition from an $A$-like phase to a $B$-like phase is marked by a discontinuous change in the magnetization. This was first seen by Barker et al $\underline{60}$ in a $98 \%$ aerogel and subsequently by many other groups! 142958616263 Additionally the frequency spectrum in most NMR experiments is substantially broadened inhomogeneously owing to a distribution in texture of the order parameter whose orientation relative to the magnetic field can generate a wide range of frequency shifts, $\Delta \omega$, which can be expressed ${ }^{38}$ for pure ${ }^{3} \mathrm{He}$ as,

$$
\begin{gathered}
\omega^{2}=\omega_{0}^{2}+F_{A, B}(\theta, \phi) \Omega_{A, B}^{2} \\
\Delta \omega \equiv \omega_{0}-\omega \simeq F_{A, B}(\theta, \phi) \frac{\Omega_{A, B}^{2}}{2 \omega_{0}},
\end{gathered}
$$

where $\Omega_{A, B}$ is the longitudinal resonance frequency for the $A$ or $B$-phase, $\omega_{0}$ is the NMR Larmor frequency, and $F_{A, B}(\theta, \phi) \leq 1$ depends on the NMR tip angle, $\phi$, and the orientation of the order parameter, $\theta$. However, the existence of negative shifts for $\phi \approx 0$ (the cw-NMR case for example,) even if only in some portions of the spectrum, is a direct indication $\sqrt{60}$ of a phase other than the isotropic phase. The isotropic phase can only have positive shifts;

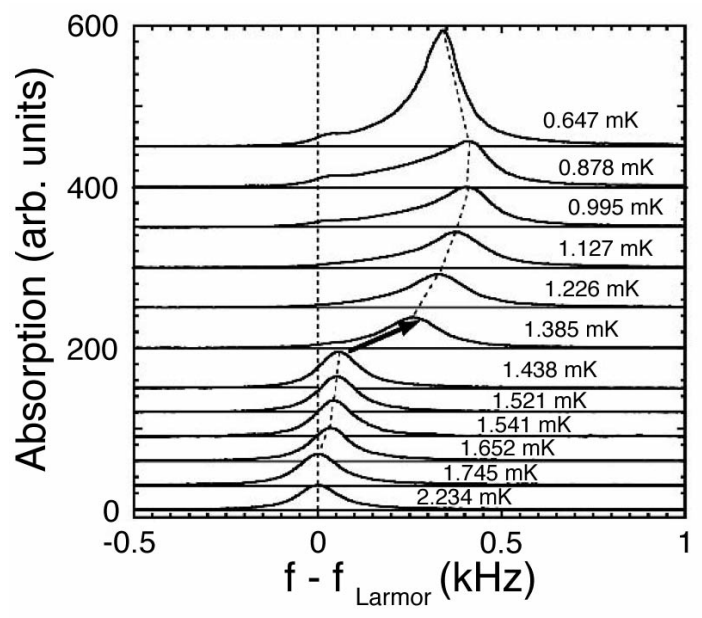

FIG. 10: Temperature dependence of the NMR spectra passing from the normal to $A$-like phase and then to the $B$-like phase for a $97.5 \%$ aerogel, uncorrected for surface solid ${ }^{3} \mathrm{He}$, obtained by Nakagawa et al! ${ }^{62}$ If maximum shifts in the NMR spectra, allowing for the normal state lineshape, are identified with the longitudinal resonance frequency, $\Omega_{A, B}$, i.e. $F_{A}=1, F_{B}=0.8$ in Eq. 18 , then the relative magnitude of the shifts in the spectra appear to be roughly consistent with a transition from the axial state to the isotropic state, Eq. 20, provided the angular momentum vector is perpendicular to the field, $\ell \perp H$. (See the discussion by Elbs et al ${ }^{[17}$ ) 


\begin{tabular}{|c|c|c|c|c|c|c|c|c|c|c|}
\hline \multirow[b]{2}{*}{$P$} & \multicolumn{5}{|c|}{ Pure ${ }^{3} \mathrm{He}$} & \multicolumn{5}{|c|}{${ }^{3} \mathrm{He}$ in $98 \%$ aerogel } \\
\hline & $\frac{\beta_{1}}{\beta_{0}}$ & $\frac{\beta_{2}}{\beta_{0}}$ & $\frac{\beta_{3}}{\beta_{0}}$ & $\frac{\beta_{4}}{\beta_{0}}$ & $\frac{\beta_{5}}{\beta_{0}}$ & $\frac{\beta_{1}^{a}}{\beta_{0}^{a}}$ & $\frac{\beta_{2}^{a}}{\beta_{0}^{a}}$ & $\frac{\beta_{3}^{a}}{\beta_{0}^{a}}$ & $\frac{\beta_{4}^{a}}{\beta_{0}^{a}}$ & $\frac{\beta_{5}^{a}}{\beta_{0}^{a}}$ \\
\hline w.c. & -1 & 2 & 2 & 2 & -2 & -1 & 2 & 2 & 2 & -2 \\
\hline 0 & -0.97 & 1.89 & 2.10 & 1.85 & -1.84 & & & & & \\
\hline 1 & -0.97 & 1.94 & 1.96 & 1.72 & -1.82 & & & & & \\
\hline 2 & -0.97 & 1.96 & 1.86 & 1.63 & -1.82 & & & & & \\
\hline 3 & -0.98 & 1.99 & 1.81 & 1.56 & -1.82 & & & & & \\
\hline 4 & -0.98 & 1.99 & 1.76 & 1.52 & -1.82 & & & & & \\
\hline 5 & -0.98 & 1.99 & 1.74 & 1.48 & -1.81 & -0.05 & 0.15 & 0.10 & 0.15 & -0.15 \\
\hline 6 & -0.98 & 1.99 & 1.72 & 1.46 & -1.81 & -0.20 & 0.51 & 0.36 & 0.48 & -0.50 \\
\hline 7 & -0.98 & 1.98 & 1.70 & 1.44 & -1.81 & -0.28 & 0.72 & 0.53 & 0.66 & -0.70 \\
\hline 8 & -0.98 & 1.98 & 1.70 & 1.42 & -1.81 & -0.35 & 0.87 & 0.66 & 0.78 & -0.84 \\
\hline 9 & -0.99 & 1.98 & 1.69 & 1.41 & -1.81 & -0.41 & 0.99 & 0.75 & 0.87 & -0.95 \\
\hline 10 & -0.99 & 1.97 & 1.69 & 1.40 & -1.85 & -0.45 & 1.08 & 0.83 & 0.94 & -1.05 \\
\hline 11 & -0.99 & 1.97 & 1.70 & 1.39 & -1.85 & -0.49 & 1.15 & 0.90 & 0.99 & -1.12 \\
\hline 12 & -0.99 & 1.96 & 1.69 & 1.39 & -1.85 & -0.52 & 1.21 & 0.96 & 1.03 & -1.17 \\
\hline 13 & -0.99 & 1.95 & 1.69 & 1.39 & -1.85 & -0.55 & 1.26 & 1.01 & 1.06 & -1.22 \\
\hline 14 & -1.00 & 1.95 & 1.70 & 1.38 & -1.85 & -0.58 & 1.30 & 1.05 & 1.09 & -1.27 \\
\hline 15 & -1.00 & 1.95 & 1.72 & 1.35 & -1.89 & -0.60 & 1.34 & 1.10 & 1.10 & -1.32 \\
\hline 16 & -1.00 & 1.95 & 1.73 & 1.34 & -1.89 & -0.62 & 1.38 & 1.13 & 1.12 & -1.35 \\
\hline 17 & -1.00 & 1.94 & 1.72 & 1.33 & -1.89 & -0.64 & 1.40 & 1.16 & 1.13 & -1.38 \\
\hline 18 & -1.00 & 1.94 & 1.73 & 1.32 & -1.89 & -0.66 & 1.43 & 1.19 & 1.14 & -1.41 \\
\hline 19 & -1.00 & 1.93 & 1.72 & 1.33 & -1.89 & -0.67 & 1.45 & 1.21 & 1.16 & -1.43 \\
\hline 20 & -1.01 & 1.94 & 1.74 & 1.31 & -1.93 & -0.69 & 1.48 & 1.24 & 1.16 & -1.47 \\
\hline 21 & -1.01 & 1.94 & 1.74 & 1.29 & -1.93 & -0.71 & 1.50 & 1.26 & 1.16 & -1.49 \\
\hline 22 & -1.01 & 1.93 & 1.74 & 1.29 & -1.93 & -0.72 & 1.51 & 1.28 & 1.17 & -1.51 \\
\hline 23 & -1.01 & 1.93 & 1.74 & 1.29 & -1.93 & -0.73 & 1.53 & 1.30 & 1.18 & -1.53 \\
\hline 24 & -1.01 & 1.93 & 1.74 & 1.28 & -1.93 & -0.74 & 1.54 & 1.32 & 1.18 & -1.54 \\
\hline 25 & -1.01 & 1.93 & 1.74 & 1.28 & -1.97 & -0.75 & 1.56 & 1.33 & 1.18 & -1.58 \\
\hline 26 & -1.02 & 1.93 & 1.73 & 1.27 & -1.97 & -0.76 & 1.57 & 1.34 & 1.18 & -1.60 \\
\hline 27 & -1.02 & 1.93 & 1.74 & 1.26 & -1.97 & -0.77 & 1.58 & 1.36 & 1.18 & -1.61 \\
\hline 28 & -1.02 & 1.93 & 1.73 & 1.26 & -1.97 & -0.78 & 1.60 & 1.37 & 1.19 & -1.62 \\
\hline 29 & -1.02 & 1.93 & 1.73 & 1.26 & -1.97 & -0.78 & 1.61 & 1.38 & 1.19 & -1.63 \\
\hline 30 & -1.02 & 1.93 & 1.72 & 1.26 & -2.01 & -0.79 & 1.62 & 1.38 & 1.19 & -1.67 \\
\hline 31 & -1.03 & 1.93 & 1.73 & 1.25 & -2.01 & -0.80 & 1.62 & 1.40 & 1.19 & -1.68 \\
\hline 32 & -1.03 & 1.93 & 1.73 & 1.25 & -2.01 & -0.81 & 1.63 & 1.40 & 1.19 & -1.68 \\
\hline 33 & -1.03 & 1.93 & 1.73 & 1.25 & -2.01 & -0.81 & 1.63 & 1.41 & 1.19 & -1.69 \\
\hline 34 & -1.03 & 1.93 & 1.73 & 1.25 & -2.01 & -0.82 & 1.64 & 1.42 & 1.20 & -1.70 \\
\hline
\end{tabular}

TABLE I: $\beta_{i}$ 's for pure superfluid ${ }^{3} \mathrm{He}$, left side, and superfluid ${ }^{3} \mathrm{He}$ in aerogel in IISM with $\lambda=150 \mathrm{~nm}$ and $\xi_{a}=40 \mathrm{~nm}$, right side. These results are taken from Choi et al. ${ }^{39}$.

so negative shifts can be interpreted as an indication of an $A$-like phase, a point that was emphasized by Barker et al ${ }^{[60}$ For the $\operatorname{axial}(A)$ and isotropic $(B)$ states we know further that,

$$
\frac{\Omega_{B}^{2}}{\Omega_{A}^{2}}=\frac{5}{2}\left(\frac{\chi_{A}}{\chi_{B}}\right) \frac{\Delta_{B}^{2}}{\Delta_{A}^{2}}
$$

If we identify $B$ and $A$-like phases, respectively with the isotropic and axial states, then close to $T_{c a}$ where $\Delta_{A} \approx \Delta_{B}$ (since $\beta_{A} \approx \beta_{B}$ from table I) and $\chi_{A}=\chi_{B}$, we expect the largest frequency shifts of the $B$-like phase to be two times larger than for the $A$-like phase. The ratio of frequency shifts observed for the two phases in a $97.5 \%$ aerogel by Nakagawa et al. $\frac{62}{62}$ reproduced in Fig. 10, appear to be consistent with this behavior provided $\ell \perp H$. Baumgardner and Osherofl ${ }^{64}$ have made similar arguments to determine the free energy of $A$-like and $B$-phases in a $99.3 \%$ aerogel, and Dmitriev et al. ${ }^{16}$ have also analyzed specta for a $98 \%$ aerogel to obtain the relative magnitudes of the longitudinal resonance frequencies in the two phases. In both cases the spectra are complex owing to a distribution of textures and, in the latter case, coexistence of these two phases in different parts of their aerogel sample. Direct observation of the longitudinal resonance frequency has also been reported by this group in both phases ${ }^{65}$ as well as observation ${ }^{66}$ of a homogeneous precession domain (HPD) in the aerogel $B$-phase. Sato et al ${ }^{[15}$ have observed HPD in an ESPstate of superfluid ${ }^{3} \mathrm{He}$ for the first time using aerogel. This was obtained in the $A$-like phase in a sample that was axially anisotropic with symmetry axis parallel to the magnetic field. This arrangement has maximum dipole energy and leads to maximal negative shifts in the spectrum; however, the tip angle dependence of the dipole energy provides a favorable situation for the formation of a HPD in this case, not realized in pure ${ }^{3} \mathrm{He}$.

Complementary to the NMR methods, which require an applied field, acoustic measurements in zero applied 


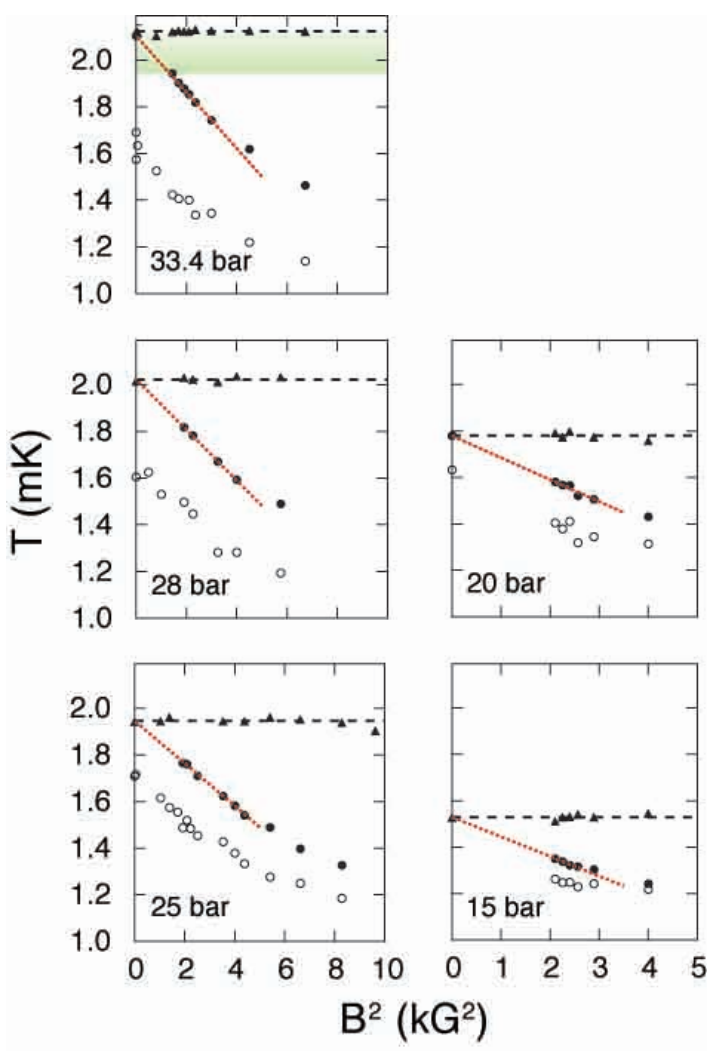

FIG. 11: Phase diagram for a $98 \%$ aerogel superfluid in a magnetic field from Gervais et al ${ }^{21}$ An equal-spin pairing-state like the $A$-phase was identified from the independence of its transition temperature on field. This phase destabilizes the dominant $B$-phase with increasing magnetic field at all pressures studied. The temperature interval for metastability of the $A$-like phase (open circles) appears to be independent of field, on which basis Gervais et al. inferred that the window of stable $A$-like phase in zero magnetic field was very small. This argument is based on the assumption that the $B$ to $A$-like phase transition does not superheat, as is the case for pure ${ }^{3} \mathrm{He}$, and the fact that the zero-field extrapolation of the warming transition points directly toward $T_{c a}$, as is shown in this figure.

field $20|21| 67$ can identify phase transitions with excellent resolution. Then one can determine which of those transitions are associated with $A$-like or $B$-like phases by applying a field, the isotropic phase being strongly suppressed by field as compared to an ESP-phase. Based on such methods Gervais et al ${ }^{21}$ concluded that the entire pressure-temperature, superfluid region in zero applied magnetic field for a $98 \%$ aerogel was a $B$-like phase, with the possible exception of a narrow region just below $T_{c a}$, $\approx 20 \mu \mathrm{K}$ wide. In this $B$-like phase Dmitriev et al ${ }^{68} \mathrm{de}$ de termined a Leggett angle equal to $104^{\circ}$ from pulsed NMR measurements as a function of pulse tip-angle, thereby confirming that the $B$-like phase was the isotropic state similar to pure superfluid ${ }^{3} \mathrm{He}$. In the following we will refer to this phase simply as the $B$-phase.

Using transverse acoustic impedance, Gervais et al ${ }^{21}$

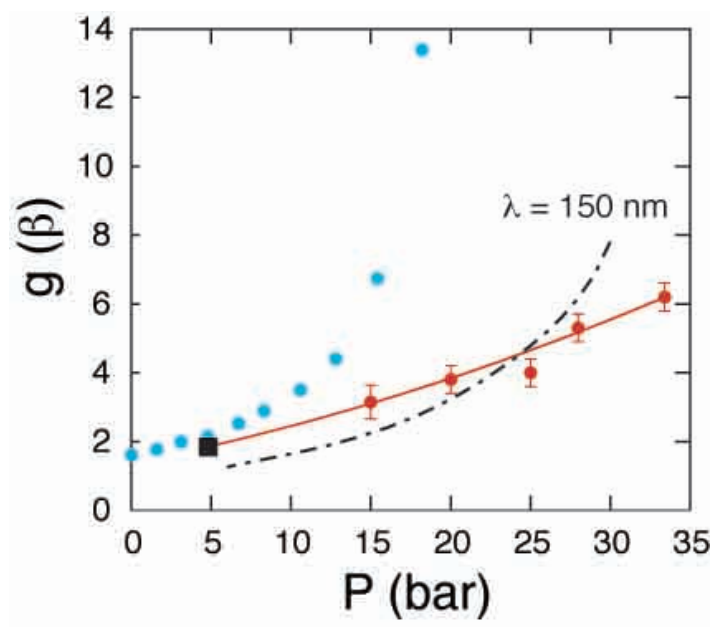

FIG. 12: The quadratic field suppression of the $B$-phase in a $98 \%$ aerogel from Gervais et al. ${ }^{21}$ These data are taken from the phase diagram measurements in Fig. 11. The same factor $g(\beta)$ was obtained for either warming or cooling and is shown here compared to their fit (dotdashed curve) to the HISM giving $\lambda=150 \mathrm{~nm}$. The black square is taken from Brussard et al ${ }^{69}$ Pure ${ }^{3} \mathrm{He}$ is shown by solid (blue) circles ${ }^{46}$ and the solid (red) curve is a guide to the eye.

measured the magnetic field suppression of the $B$-phase for several representative pressures from 15 to 33.4 bar, Fig. 11. On cooling in a magnetic field the metastable $A$-like phase was first nucleated at a field-independent value, $T_{c a}$, in the range from zero-field to $0.5 \mathrm{~T}$. The field dependence for transitions from $A$-like to $B$-phases on cooling was quadratic in field. On warming the $B$ to $A$-like transition was displaced to a higher temperature but with the same quadratic field dependence. It is likely that the magnetic field dependence of the equilibrium transition is the same as found in these warming and cooling experiments since those data are parallel to one another. Gervais et al. determined $g(\beta)$ from their analysis of this field dependence in terms of Eq. 11 and 12. The HISM can account for the observed behavior with a scattering mean free path of $150 \mathrm{~nm}$, Fig. 12. Brussard et al ${ }^{69}$ determined the field dependence of the $A$-like to $B$-transition at 4.8 bar with an oscillating aerogel paddle and these results are consistent, shown by the black square in Fig. 12.

The $A$-like phase is known to be an ESP-phase even if it is not well-established to which $p$-wave state this corresponds. For sufficiently large magnetic fields an ESP-state should split into $A_{1}$-like and $A_{2}$-like transitions owing to particle-hole asymmetry. Barmadze and Kharadze ${ }^{70}$ and Sauls and Sharma $a^{\sqrt{52}}$ have considered this problem for superfluid ${ }^{3} \mathrm{He}$ in aerogel taking into account the exchange coupling between the paramagnetic surface solid ${ }^{3} \mathrm{He}$ and the superfluid which will either decrease or increase the splitting depending on whether the interaction is antiferromagnetic or ferromagnetic. Since there did not appear to be evidence for a splitting in 

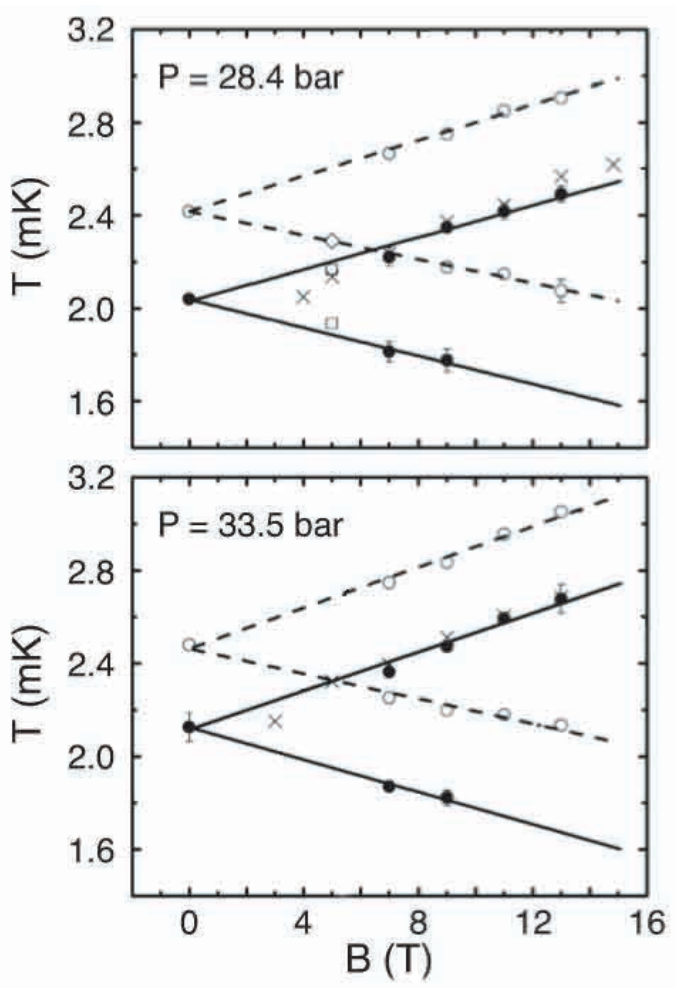

FIG. 13: Splitting of the $A_{1}-A_{2}$ transition at high magnetic field for superfluid ${ }^{3} \mathrm{He}$ in a $98 \%$ aerogel (solid circles and crosses) and in pure ${ }^{3} \mathrm{He}$ (open circles) at two pressures by Choi et al ${ }^{71}$ The linear slopes with magnetic field are rather similar for pure and aerogel superfluids.

experiments ${ }^{21}$ up to $0.8 \mathrm{~T}$ this suggested that the coupling was antiferromagnetic. But higher field experiments are required, as well as measurements with ${ }^{4} \mathrm{He}$ preplating, to reach a firm conclusion. In this spirit Choi et al $!^{71}$ extended the field range of Gervais et al. ${ }^{21}$ to $H=14 \mathrm{~T}$ using the same aerogel sample, and their results are reproduced in Fig. 13. Transverse sound techniques were used and the $A_{1}-A_{2}$ splitting was clearly identified in both pure and aerogel superfluids. The splitting ratio, $r$, was found to be similar for the two cases. Owing to experimental conditions, the accuracy of the transition temperatures measured was not sufficient at low magnetic fields to make a quantitative comparison with the exchange models. $\frac{52170}{5}$

\section{B. Specific Heat, Thermal Conductivity, Gaplessness}

The most direct determination of the magntitude of the order parameter is found from the specific heat jump, a measurement that can be interpreted in terms of the GL-theory and which determines corresponding values of the $\beta$-parameters. Specific heat measurements were performed by Choi et al. ${ }^{[55}$ as shown in Fig. 14 and 15 . In this experiment an adiabatic calorimetric method was

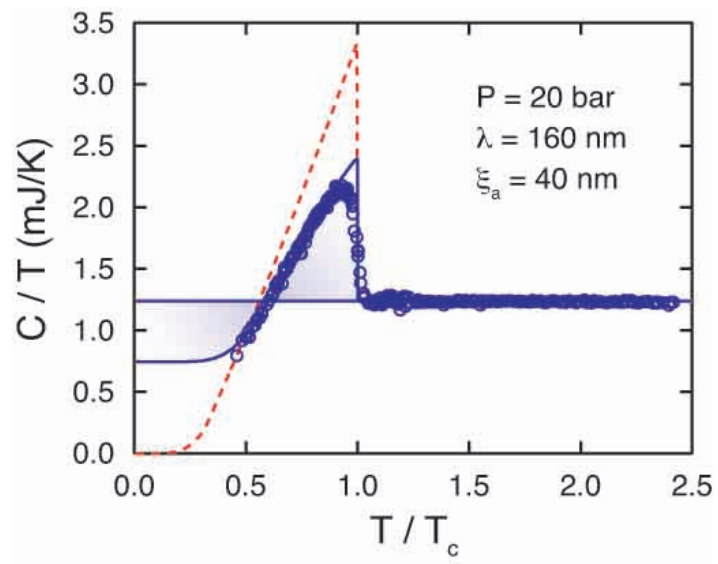

FIG. 14: Specific heat jump as a function of temperature for the $B$-phase of a $98 \%$ aerogel at $P=20$ bar. Fitting to the data gives the scattering parameters of mean-freepath $\lambda=160 \mathrm{~nm}$ and $\xi_{a}=35 \mathrm{~nm}$ from the IISM of Sauls and Sharma. ${ }^{[52}$ The specific heat for pure ${ }^{3} \mathrm{He}$ is shown for comparison as a dashed curve.

used, hampered in part by the small volume of the aerogel sample, $1.1 \mathrm{~cm}^{3}$, and a background attributed to paramagnetic solid on the aerogel surface that was subtracted. On the other hand, very low heat leak, $\approx 0.1 \mathrm{nW}$, and high resolution thermometry gave accurate measurements, particularly for the specific heat jump. A comparison of the jump for pure and aerogel superfluids is made in Fig. 15. Good agreement was found with the predictions of the theory as a function of pressure, leading to a best fit overall for $\lambda=160 \mathrm{~nm}$. The distinction between HISM and IISM theories is marginal in this case. Ad-

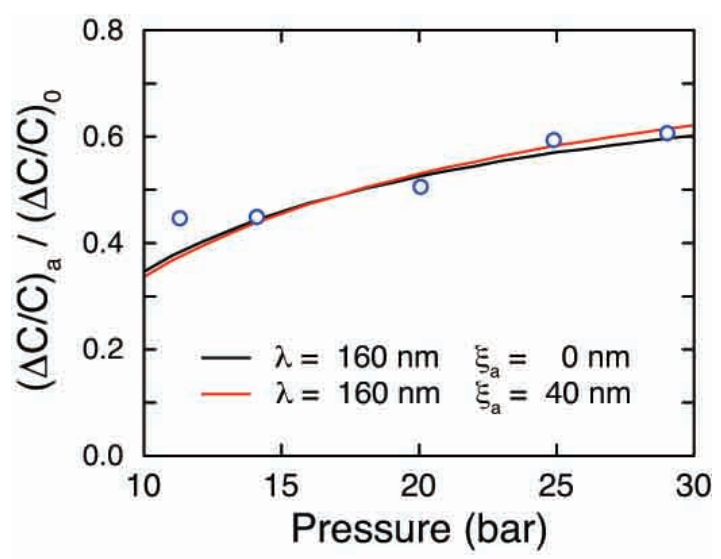

FIG. 15: Specific heat jump as a function of pressure for the $B$-phase of a $98 \%$ aerogel from Choi et al.$^{35}$ Fitting to the data gives the scattering parameters for the IISM of Sauls and Sharma ${ }^{52}$ of mean-free-path $\lambda=160 \mathrm{~nm}$ and $\xi_{a}=40 \mathrm{~nm}$. The fits are not sensitive to the correlation length, $\xi_{a}$ as shown here, in contrast to the transition temperature, Fig. 1 and 8. Additionally, there is only a small difference between $A$ - and $B$-phases although this is not shown here. 39 


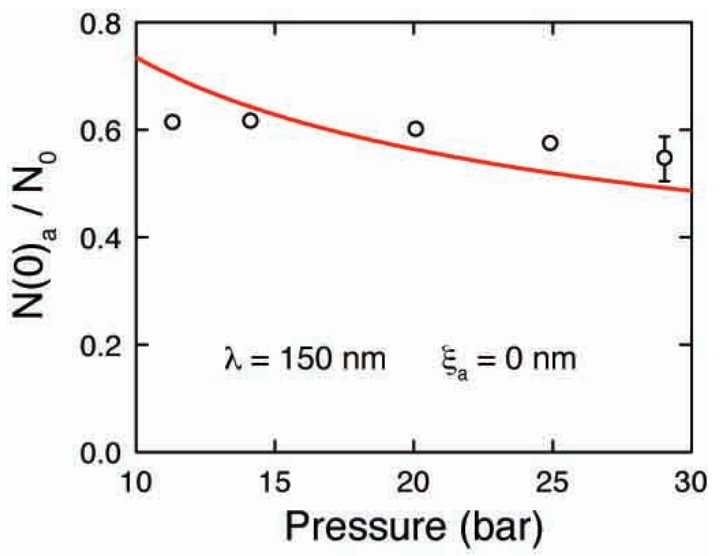

FIG. 16: The density of states (DOS) at the Fermi energy inferred from specific heat measurements of Choi et al. ${ }^{35}$ compared with calculations from the HISM. The nonzero spectral weight at the Fermi energy is attributed to overlapping bound states. This behavior is a direct indication of gapless superfluidity and has been observed and predicted for the thermal conductivity, ${ }^{[56 / 73}$ and the magnetic susceptibility $\underline{57 / 60}$

ditional calculations ${ }^{72}$ for the specific heat jump within the context of these models for $A$ and $B$-phases indicate that they are close to one another ( 5 to $10 \%$, depending on pressure) although $\Delta C_{B a}$ is always larger than $\Delta C_{A a}$, consistent with the equilibrium phase being the $B$-phase. A comparison of the order parameter suppression and that of the superfluid transition temperature for this $98 \%$ aerogel is plotted in Fig. 8.

In addition to the specific heat jump, Choi et al! $\frac{35}{\mathrm{de}} \mathrm{d}$ termined the temperature dependence of the specific heat and in particular accurate measurements of the temperature derivative of the specific heat just below $T_{c a}$. They argued on the basis of the third law of thermodynamics that entropy should be conserved, meaning that the two shaded areas in Fig. 14 must be equal and consequently if the specific heat is a monotonic function of temperature that there is a non-zero intercept of $C_{B a} / T$ at $T=0$. This observation means that there is a nonzero and significant density of states (DOS) at the Fermi energy, $E_{F} \equiv 0$. This behavior is in contrast to pure superfluid ${ }^{3} \mathrm{He}$ where the DOS is strictly zero there. Theoretically it is expected $\frac{5357}{5}$ that a broad band of Andreev bound states associated with the aerogel impurity in ${ }^{3} \mathrm{He}$ will transfer spectral weight in the DOS from the coherence peaks above $\Delta$ to zero energy, leading to gapless behavior. With reduction of pressure the DOS band develops, eventually reaching a value close to that of the normal state, in which case the specific heat on passing from the normal to the superfluid becomes featureless. It is also clear that the development of this band of states obscures the distinction between different possible $p$-wave states. Consequently, the presence of large concentrations of impurities in an unconventional superfluid or superconductor will substantially affect the low temperature thermodynamic behavior obscuring identification of the symmetry of the superfluid or superconducting state in these measurements. The observation of non-zero values of the DOS at low energy from the specific heat is plotted as a function of pressure in Fig. 16 and compared with the calculations of Sharma and Sauls $\underline{53}$

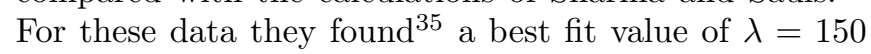
nm. Evidence for gapless behavior can also be found in the low temperature magnetic susceptibility $18|30| 57$ and the thermal conductivity ${ }^{[3]}$ In the latter case the thermal conductance indicates that a gap in the $B$-phase DOS returns for pressures in excess of about 10 bar, in contrast to the interpretation of gapless behavior at all pressures from the specific heat measurements made by Choi et al.

Thermal conductivity measurements have been performed by the Lancaster group, 2873 for which the high temperature superfluid behavior is presented in Fig. 17, showing that there is a weak anomaly at the transition temperature. Behavior in the normal fluid and in the superfluid phases has been analyzed by Sharma and Sauls $\sqrt{56}$ from which $\lambda=205 \mathrm{~nm}$ was determined, somewhat larger than the trend from other experiments. It is possible that the thermal boundary resistance between the aerogel and pure ${ }^{3}$ He superfluids might play a significant role.

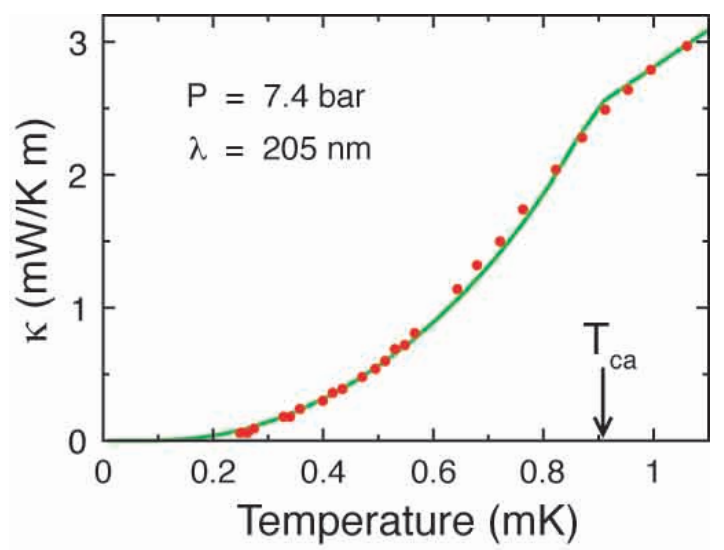

FIG. 17: Thermal conductivity data at 7.4 bar from Fisher et al..$^{28}$ analyzed $\sqrt{56}$ in terms of the HISM theory giving a value of $\lambda=205 \mathrm{~nm}$.

\section{NMR and Magnetization}

The magnetization measurements9|29|30|58|60 consistently indicate a metastable ESP-phase and a $B$-phase with a suppressed order parameter. Calculations have been performed by Sharma and Sauls $\sqrt{56}$ and Mineev $\sqrt{57}$ for which a representative example of data is shown in Fig. 18 from Barker et al ${ }^{60}$ A value of $\lambda=140 \mathrm{~nm}$ fits the data best and the distinction between HISM and IISM is not discernible. These experiments were performed with ${ }^{4}$ He preplating.

In the context of identification of the superfluid state we have discussed the NMR frequency shifts and spectra 
expected for the $A$ and $B$-phases. In some cases, such as the $99.3 \%$ aerogel samples of Baumgardner et al.58 the spectrum is very broad; in other cases ${ }^{8114|17| 63}$ much less so. In the first NMR experiments, Sprague et al. . took data only on warming. It is not obvious how to reconcile their measurements with their claim that the experiments were conducted in the (supercooled) $A$-like phase since the extent of supercooling reported by other workers2012167 is typically a factor of two less than the range of supercooling that would be required by Sprague et al. On the other hand, they measured the magnetization with sufficient accuracy to identify an ESP-phase. Later analysis by Haard, ${ }^{25}$ based on independent measurements of the magnetization, confirmed the earlier conclusions. Additionally, Sprague et al. found very little inhomogeneous broadening of their spectra, in contrast to what has been found by most groups to be characteristic of the $B$-phase. Finally, the amplitude of the order parameter inferred from frequency shifts measured by Sprague et al. are in excellent agreement with specific heat jump measurements as displayed in Fig. 8 and 19 . If the data of Sprague et al. were indeed in the $B$-phase, the texture of the order parameter would necessarily have to be dipole-unlocked, but homogeneously so, in order to have a well-defined value of $F(\theta, \phi)$ in Eq. 18. This seems rather unlikely although it cannot be ruled out. In summary, we believe that Sprague et al. observed extensive supercooling of the metastable $A$-like phase in those early NMR experiments. We have recently inspected the aerogel sample they used with optical birefringence methods and found that it was homogeneous and isotropic ${ }^{22}$ Furthermore, the aerogel sample was well separated $(\approx 0.2$ $\mathrm{mm}$ ) from the walls eliminating the possibility of stress accumulation from differential contraction during cooling. These aspects may account for the homogeneous NMR spectra and large supercooling.

\section{Acoustics and Mechanical Response}

Low frequency sound ${ }^{67 / 74}$ and high frequency ultrasound propagation have been observed $21 / 27 / 75$ in superfluid ${ }^{3} \mathrm{He}$ in aerogels and these techniques, combined with transverse acoustic impedance methods, $\stackrel{20121}{2}$ have been used to identify phase transitions as well as the superfluid density. Calculations ${ }^{75 / 76}$ have focused on the coupling between the silica framework and the ${ }^{3} \mathrm{He}$ modes and yielded excellent agreement with experiment including the collision drag effect.

The mechanical dynamics of aerogel samples affixed to a wire resonator have also been used by the Lancaster group $\frac{69177}{6}$ to investigate the phase diagram and have been exploited to determine the transitions between phases and anisotropy in the superfluid density which may be relevant in distinguishing between various possible candidates for the metastable $A$-like phase.

At this time there is no evidence of a propagating high frequency shear mode in superfluid ${ }^{3} \mathrm{He}$ in aerogel and

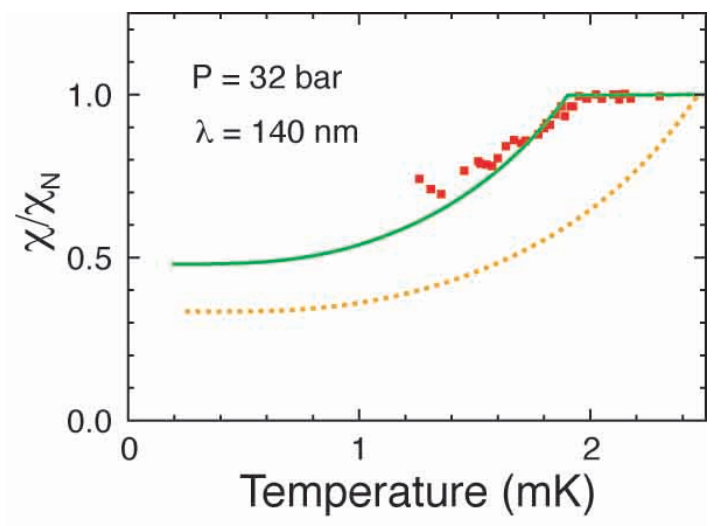

FIG. 18: Magnetic susceptibility measurements in the $B$ phase of superfluid ${ }^{3} \mathrm{He}$ in a $98 \%$ aerogel at $P=32$ bar from NMR by Barker et al ${ }^{60}$ analyzed by Sharma and Sauls ${ }^{\sqrt{53}}$ in terms of the HISM. A value of $\lambda=140 \mathrm{~nm}$ was found although the temperature range of the experiment is not extensive. The distinction between HISM and IISM is rather slight. The susceptibility for pure ${ }^{3} \mathrm{He}$ is shown as a dotted curve. Similar work has been reported by the Grenoble group ${ }^{30}$ for a $98 \%$ aerogel where they consistently found $\lambda=140$ for data in the range $P=17$ to 30 bar.

there is no evidence from acoustics of order parameter collective modes ${ }^{27 / 75}$ nor an anomaly in attenuation associated with a threshold for pair breaking. These features have been extensively explored in the pure superfluid phases ${ }^{78}$

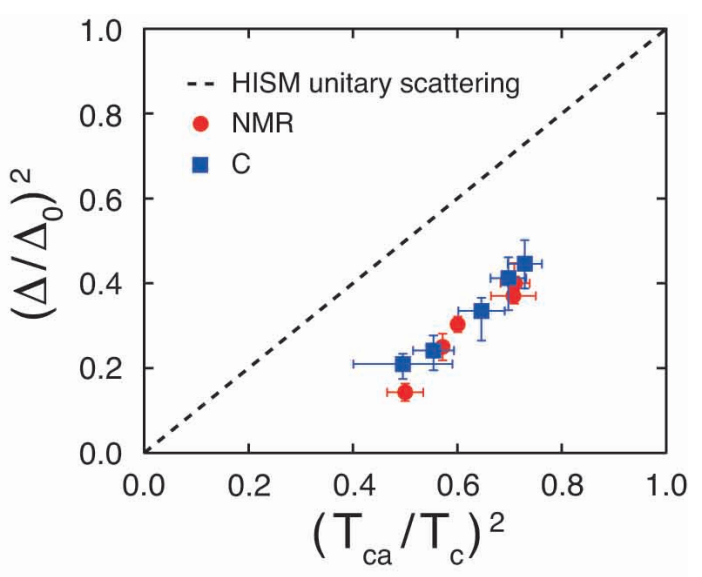

FIG. 19: A direct comparison of the order parameter suppression relative to the transition temperature is shown for $98 \%$ aerogel, compared to the HISM with unitary scattering shown as a dashed line. The excellent agreement between the specific heat data ${ }^{35}$ and the frequency shift measurements $s^{8 \mid 25}$ give support for the correctness of the phase identification made by Sprague et al. ${ }^{8}$ 


\section{E. Summary of Comparison of the IISM with Experiment}

In the previous sections we have discussed applications of the scattering models to the analysis of experiments on superfluid ${ }^{3} \mathrm{He}$ in aerogel. These give a remarkably consistent picture. The scattering model parameters which we have found to be typical of a $98 \%$ porosity aerogel are: $\lambda=150 \mathrm{~nm}, \xi_{a}=40 \mathrm{~nm}$ assuming unitary scattering, $\delta_{0}=\pi / 2$. These parameters for the IISM can account for most experiments including the phase diagram, specific heat, thermal conductivity, magnetic suscpetibility, superfluid density, NMR frequency shifts, and the high field $A_{1}-A_{2}$ splitting of the normal to superfluid transition. With the five $\beta_{i}$ 's for pure superfluid given in Table I, we use the Sauls and Sharma IISM-approach to define a modified depairing parameter and calculate the $\beta_{i}$ 's for the impure superfluid. The results of the calculation of $\beta_{i}^{a} / \beta_{0}^{a}$ are tabulated in the last five columns of Table I. For these parameters the superfluid state is not stable below a pressure of 5 bar as reported by Matsumoto et $a l{ }^{19} \mathrm{~A}$ direct consequence of the modification of $\beta_{i}$ 's, according to the scattering models is the enhancement of relative stability of the $B$-phase with respect to the $A$-phase over the entire pressure range below the melting pressure of ${ }^{3} \mathrm{He}$. Consequently, the existence of a metastable anisotropic phase suggests that the form of the free energy in Eq. 2 is incomplete.

\section{METASTABLE $A$-LIKE PHASE}

Superfluid ${ }^{3} \mathrm{He}$ in aerogel has a metastable phase that has been clearly observed $8|20| 21 / 58 / 60|67 / 69| 77 \mid$ in various samples appearing on cooling below $T_{c a}$ in a magnetic field. It is natural to expect that a window of $A$-phase might open up in a magnetic field and that this phase might supercool substantially, possibly even at low pressures. The exact nature of this phase remains an open question, although NMR experiments have shown that the magnetic susceptibility is unchanged from the normal state indicating that it is an ESP-state, like the $A$ phase $\sqrt{860}$ It is significant that Gervais et al. showed ${ }^{20}$ that the same phenomenon of metastability of an $A$-like phase occurs in experiments in zero applied field; evidently the mechanism for nucleation and the corresponding metastability is independent of magnetic field. Additional work at various pressures in zero applied field lead to the same conclusions. ${ }^{2167}$ Based on these experimental results and from the theoretical suggestions of Thuneberg et al $!^{34}$, Vicente et al ${ }^{10}$ proposed that anisotropic scattering might be the origin for stability of the $A$-like phase. However, within the context of the free energy expansion, Eq. 2, the question of stability of any ESP-state with repect to the aerogel $B$-phase relies on an understanding of the appropriate $\beta$-parameters, at least in the Ginzburg-Landau limit close to $T_{c a}$. The relative stability of axial and isotropic phases can be determined for IISM from the $\beta$-parameter combinations taken from Table I that give $\Delta C_{A a}$ and $\Delta C_{B a}$, Eq. 9 and 10. The two phases are thermodynamically so close that they will be difficult to distinguish directly $(5 \sim 10 \%)$ in specific heat measurements ${ }^{[72}$ Nonetheless, as we have mentioned previously, it is likely that anisotropic scattering or some other modification of the theory will be required to understand this interesting phenomenon.

Volovik ${ }^{79}$ has argued that the axial state in the presence of quenched anisotropic disorder cannot exist as a spatially homogeneous superfluid. So the axial state would not have long range orientational order, a state which Volovik has called a superfluid glass or a LarkinImry-Ma state ${ }^{80}$ With a different approach, Fomin 81 has argued that there are other $p$-wave pairing states which are also ESP-states but do not suffer from the same difficulty, and that these might be candidates for the metastable aerogel phase. Such phases would be robust in the presence of anisotropic scattering, meaning that $A_{\mu i} A_{\mu j}^{*}+A_{\mu j} A_{\mu i}^{*} \propto \delta_{i j}$ where $\delta_{i j}$ is a Kronecker delta 81 NMR experiments have been performed on ${ }^{3} \mathrm{He}$ in $97.5 \%$ aerogel which support the view that the metastable $A$-like phase is in fact a robust state, $\frac{82}{2}$ but other measurements $\frac{1665}{165}$ appear to have inconsistencies with this interpretation.

If we assume that the free energy expansion of Eq. 2 is correct then we can use the $\beta_{i}$ 's from Table I to determine the asymmetry ratio predicted for the robust and axial states and compare with experiment ${ }^{71}$ For the axial state the ratio is expressed in terms of the $\beta_{i}$ 's given by Eq. 13 for which we find $r$ between 1.0 and 1.5, increasing with increasing pressure consistent with the experimental results $r_{a} \gtrsim 1.0 .71$ In the case of the robust state ${ }^{35181}$,

$$
r_{R}=\frac{\beta_{15}}{\beta_{12345}+4 \beta_{2}+4 \beta_{245}} .
$$

With the values of the $\beta_{i}$ 's from the table, the asymmetry ratio $r_{R}$ is found to be $\sim 0.2$, considerably smaller than what was observed.

\section{ANISOTROPIC AEROGEL}

There are two important issues regarding anisotropy. First, according to the scattering model ${ }^{34}$ isotropic elastic scattering stabilizes the isotropic state and anisotropic scattering favors an anisotropic state. Secondly, all experiments are consistent with the formation of a metastable anisotropic phase (there is only one isotropic $p$-wave state) which somehow nucleates on cooling through $T_{c a}$. To reconcile these points Vicente et al 10 proposed that the local anisotropy inherent to the aerogel structure on the scale of $\xi_{a}$ should lead to anisotropic scattering that could nucleate an anisotropic phase. Furthermore this effect will be more prevalent for shorter coherence lengths, i.e. at higher pressure. The physical mechanism for this phenomenon is completely independent of that which stabilizes the $A$-phase in pure 
superfluid ${ }^{3}$ He i.e. strong-coupling. These authors suggested that globally imposed anisotropy might then increase the stability of the $A$-like phase. Aoyama and Ikeda ${ }^{11}$ explored this idea theoretically finding that this might indeed be the case and that one might expect a difference between samples that were axially compressed, as opposed to radially compressed; the latter possibly stabilizing the polar state in preference to the axial state. The first experiments with globally induced axial anisotropy were reported by Davis et al $!^{[12}$ where radial compression of $10 \%$ was induced by preferential shrinkage during growth and drying of the aerogel. In this work a stable region of superfluid was indeed established, although the nature of this phase has not been explored. In later work Davis et al ${ }^{13}$ studied a $17 \%$ axially compressed aerogel, also nominally $98 \%$ in porosity, using transverse acoustic impedance methods to detect the $A$-like to $B$ phase transition. This sample was also characterized by optical birefringence techniques. ${ }^{22 / 83}$ The results of the supercooling experiments are compared in Fig. 20 with those for nominally unstrained samples used by Gervais et al..$^{21}$ and Nazaretski et al.,$\sqrt{67}$ both in zero applied magnetic field. The supercooling region was approximately the same in all three cases at high pressure. But at low pressure, $P \sim 10$ bar there was significantly more supercooling observed in the axially compressed sample. Davis et al $!^{[12]}$ did not find evidence for a stable region of $A$-like phase near $T_{c a}$ in their strained sample.

Most NMR experiments report that the spectrum of ${ }^{3} \mathrm{He}$ in aerogel becomes strongly inhomogeneously broadened in the superfluid state. This phenomenon, in contrast to what is observed in similar size samples of pure ${ }^{3} \mathrm{He}$, has its origin in a distribution of orientations of the order parameter, or textures $\stackrel{[38}{\text { It }}$ is likely that the texture distributions can be attributed to ansiotropy in the aerogel. In a remarkable experiment, Kunimatsu et al!14 showed that the NMR spectrum in one sample was maxi-

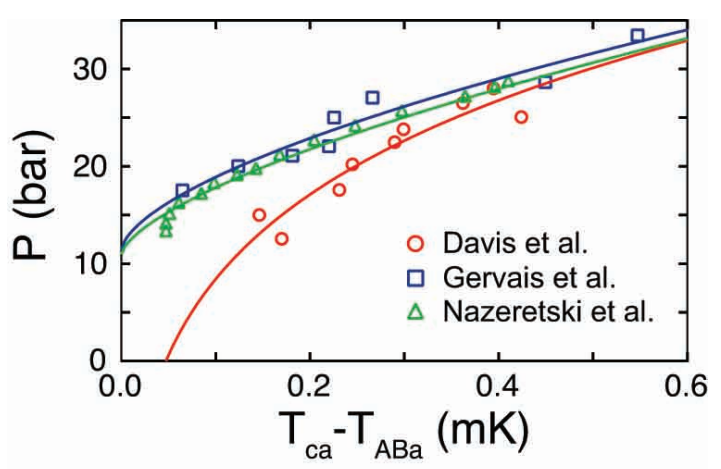

FIG. 20: Supercooling of the metastable $A$-like phase as reported for a $17 \%$ axially strained sample of $98 \%$ aerogel by Davis et al. ${ }^{[13}$ as compared with nominally unstrained samples from Gervaiset al. ${ }^{[21}$ and Nazaretski et al.$^{[67]}$ The extent of supercooling is approximately the same. Additionally, Davis et al. ${ }^{[13}$ found no evidence for a stable region of $A$-like phase near $T_{c a}$. mally shifted to negative values and they interpreted this phenomenon to alignment of the angular momentum vector, $\ell$, with the anisotropy axis and parallel to the magnetic field. The existence of significant axial anisotropy in that sample was later confirmed by optical birefringence measurments performed at Northwestern. In two other NMR experiments, Dmitriev et al! $\frac{16}{16}$ and Elbs et al! have shown unusual order parameter textures which they identified with anisotropy within the aerogel. A common feature of the two experiments is the coexistence of both $B$ and $A$-like phases for a range of temperature and clear evidence for the orientation of $\ell$ by the aerogel structure. It is interesting that the NMR spectra from the radially compressed aerogel of Elbs et al 17 are similar to the spectra obtained for unstrained samples reported by Nakagawa et al. $\underline{62}$

\section{CONCLUSIONS}

In the past 12 years, considerable experimental and theoretical work has been reported on the effects of highly porous silica aerogel on the superfluid states of ${ }^{3} \mathrm{He}$. The aerogel structure provides a distribution of point-like scattering centers that can be interpreted as impurities. In this review we have concentrated on experiments and theoretical predictions of the scattering models and we find that there is very good agreement between homogeneous scattering models and experiments except for the transition temperature. In order to account for both the transition temperature suppression and the other properties of superfluid ${ }^{3} \mathrm{He}$ some form of inhomogeneous scattering must be present. The phenomenological inhomogeneous scattering model of Sauls and Sharma meets this requirement with two parameters, the transport mean free path and the aerogel particle-particle correlation length. As a result, a consistent description in terms of these two parameters can be obtained for the phase diagram, specific heat, thermal conductivity, magnetic susceptibility, superfluid density, NMR longitudinal resonance frequency, and the high field $A_{1}-A_{2}$ splitting of the normal to superfluid transition.

Exciting work currently in progress is exploring the possibility of manipulating the superfluid state using specially prepared aerogel samples that exhibit global anisotropy with the broad view that such anisotropic materials may stabilize anisotropic superfluid states, possibly states that otherwise would not exist. It is already clear from NMR experiments that the orientation of the order parameter in the metastable $A$-like phase can be affected by global anisotropy.

There remains an unresolved problem of the nature of the metastable $A$-like phase and the related question of superfluid-phase nucleation, particularly within a very narrow region close to the transition temperature from normal to superfluid.

We acknowledge support from the National Science Foundation, DMR-0703656 and thank W.J. Gannon for 
useful discussions.

1 D.D. Osheroff, Rev. Mod. Phys. 69667 (1997).

2 J.A. Annett, Superconductivity, Superfluids and Condensates Oxford University Press, Oxford, UK (2004).

3 A.J. Leggett, Quantum Liquids. Oxford University Press, Oxford, UK (2006).

${ }^{4}$ D. Pines and M. A. Alpar, Nature 316, 27 (1985).

${ }^{5}$ K. Rajagopal and F. Wilzcek, Phys. Rev. Lett. 86, 3492 (2001).

6 A.V. Balatsky, I Vekhter, and J.X. Zhu, Rev. Mod. Phys. 78, 373 (2006).

7 J.V. Porto and J.M. Parpia, Phys. Rev. Lett. 74, 4667 (1995).

8 D.T. Sprague, T.M. Haard, J.B. Kycia, M.R. Rand, Y. Lee, P.J. Hamot and W.P. Halperin, Phys. Rev. Lett. 75, 661 (1995).

9 D.T. Sprague, T.M. Haard, J.B. Kycia, M.R. Rand, Y. Lee, P.J. Hamot, and W.P. Halperin, Phys. Rev. Lett. 77, 4568 (1996).

10 C.L. Vicente, H.C. Choi, J.S. Xia, W.P. Halperin, N. Mulders, and Y. Lee, Phys. Rev. B 72, 094519 (2005).

11 K. Aoyama and R. Ikeda, Phys. Rev. B. 73, 060504(R) (2006); K. Aoyama and R. Ikeda, J. Phys. Chem. Solids 66, 1330 (2005).

12 J.P. Davis, H. Choi, J. Pollanen, and W.P. Halperin, AIP Conf. Proc. 850, 239 (2006).

13 J.P. Davis, J. Pollanen, B. Reddy, K.R. Shirer, H. Choi, and W.P. Halperin, Phys. Rev. B 77, 140502(R) (2008).

14 T. Kunimatsu, T. Sato, K. Izumina, A. Matsubara, Y. Sasaki, M. Kubota, O. Ishikawa, T. Mizusaki, and Yu M. Bunkov, JETP Lett. 86, 216 (2007);T. Kunimatsu, K. Izumina, T. Sato, A. Matsubara, Y. Sasaki, O. Ishikawa, M. Kubota, Y.M. Bunkov, T. Mizusaki, J. Low Temp. Phys. 148, 591 (2007).

15 T. Sato, T. Kunimatsu, K. Izumina1, A. Matsubara, M. Kubota1, T. Mizusaki, and Yu. M. Bunkov, ArXive:0804.2994

16 V.V. Dmitriev, D.A. Krasnikhin, N. Mulders, V.V. Zavjalov, D.E. Zmeev, Pis'ma v ZhETF, 86, 681 (2007); JETP Lett. 86, 594 (2008).

17 J. Elbs, Yu.M. Bunkov, E. Collin, H. Godfrin, and G. Volovik, Phys. Rev. Lett. 100, 215304 (2008).

18 W.P. Halperin and J.A. Sauls, cond-mat/0408593

19 K. Matsumoto et al., Phys. Rev. Lett. 79, 253 (1997).

20 G. Gervais, R. Nomura, T.M. Haard, N. Mulders, W.P. Halperin, Phys. Rev. Lett. 87, 035701 (2001).

21 G. Gervais, K. Yawata, N. Mulders, W.P. Halperin, Phys. Rev. B 66, 054528 (2002).

22 J. Pollanen, K. Shirer, S. Blinstein, J.P. Davis, H. Choi, T.M. Lippman, L.B. Lurio, and W.P. Halperin, J. Non-Crystalline Solids: to appear (2008); cond-mat/ arXiv:0711.3495

23 J.V. Porto and J.M. Parpia, Phys. Rev. B 59, 14583 (1999).

24 T.M. Haard, G. Gervais, R. Nomura, and W.P. Halperin Physica B 284-288, 289 (2000).

25 T.M. Haard, Ph.D. thesis, Northwestern University, 2001, (unpublished).

26 J.F. Poco, P.R. Coronado, R.W. Pekala, L.W. Hrubesh, in:
R.F. Lobo, J.S. Beck, S.L. Suib, D.R. Corbin, M.E. Davis, L.E. Iton, S.I. Zones (Eds.), Microporous and Mesoporous Materials, MRS Symposium Proceedings, 431, Materials Research Society, Pittsburg, PA, 297 (1996).

27 R. Nomura, G. Gervais, T.M. Haard, Y. Lee, N. Mulders, W.P. Halperin, Phys. Rev. Lett. 85, 4325 (2000).

28 S.N. Fisher, A.M. Guenault, A.J. Hale, and G.R. Pickett, J. Low Temp. Phys. 126, 673 (2001).

29 Y.M. Bunkov, E. Collin, and H. Godfrin, J. Phys. Chem. Solids 66, 1325 (2005)

30 J.A. Sauls, Y.M. Bunkov, E. Collin, H. Godfrin, and P. Sharma, Phys. Rev. B 72, 024507 (2005).

31 A.A. Abrikosov and L.P. Gorkov, Sov. Phys. JETP 12, 1243 (1961).

32 T. Tsuneto, On Dirty Superconductors, Institute of Solid State Physics, University of Tokyo Technical Report No. 47, Ser. A, (1962).

33 A. I. Larkin , JETP Lett. 2, 130 (1965).

34 E.V. Thuneberg, S.K. Yip, M. Fogelström, and J.A. Sauls, Phys. Rev. Lett. 80, 2861 (1998).

${ }^{35}$ H. Choi, K. Yawata, T.M. Haard, J.P. Davis, G. Gervais, N. Mulders, P. Sharma, J.A. Sauls, and W.P. Halperin, Phys. Rev. Lett. 93, 145301(2004).

36 A.J. Leggett, Rev. Mod. Phys. 47331 (1975).

37 E.V. Thuneberg, Phys. Rev. B 36, 3583 (1987).

38 D. Volhardt amd P. Wölfle, The Superfluid Phases of Helium 3, Taylor and Francis, (1990).

${ }^{39}$ H. Choi, J.P. Davis, J. Pollanen, T.M. Haard, and W.P. Halperin, Phys. Rev. B 75, 174503 (2007).

40 D. Rainer and J.W. Serene, Phys. Rev. B 13, 4745 (1976).

41 N.A. Greaves, J. Phys. C. 9, L181 (1976).

42 J.B. Kycia, T.M. Haard, M.R. Rand, H.H. Hensley, G.F. Moores, Y. Lee, P.J. Hamot, D.T. Sprague, W.P. Halperin and E.V. Thuneberg, Phys. Rev. Lett. 72, 6864 (1994).

43 M.R. Rand, H.H. Hensley, J.B. Kycia, T.M. Haard, Y. Lee, P.J. Hamot, and W.P. Halperin, Physica B 194-196, 805 (1994).

44 M.R. Rand, Ph.D. thesis, Northwestern University, (unpublished) (1996).

45 D.S. Greywall, Phys. Rev. B 33, 7520 (1986).

46 Y.H. Tang et al., Phys. Rev. Lett. 67, 1775 (1991).

47 U.E. Israelson et al., Phys. Rev. Lett. 53, 1943 (1984).

48 J.A. Sauls and J.W. Serene, Phys. Rev. B 24, 183 (1981).

49 E.V. Thuneberg, S.K. Yip, M. Fogelström, and J.A. Sauls, ArXiv:cond-mat/9601148

50 B. Hänninen and E.V. Thuneberg, Phys. Rev. B 67, 214507 (2003).

51 E. V. Thuneberg, M. Fogelström, S. K. Yip, J. A. Sauls, R. Hänninen, and T. Setälä, in Quasiclassical methods in superconductivity and superfluidity, Verditz 96, edited by D. Rainer and J. A. Sauls (unpublished conference proceedings, 1998), p. 53; preprint archive cond-mat/9802044.

52 J.A. Sauls and P. Sharma, Phys. Rev. B 68, 224502 (2003).

53 P. Sharma and J.A. Sauls, J. Low Temp. Phys. 125, 115 (2001).

54 G. Baramidze, G. Kharadze, and G. Vachnadze, JETP Lett. 63, 107 (1996).

${ }^{55}$ G. Baramidze and G. Kharadze, J. Phys. Cond. Matter 
14, 7471 (2002).

56 P. Sharma and J.A. Sauls, Physica B 329, 313 (2003).

57 V.P. Mineev and P.L. Krotkov, Phys. Rev. B 65, 024501 (2002).

58 J.E. Baumgardner, Y. Lee, D.D Osheroff, L.W. Hrubesh, and J.F. Poco, Phys. Rev. Lett. 93, 055301 (2004).

59 H. Alles, J.J. Kaplinski, P.S. Wooton, J.D. Reppy, and J.R. Hook, Physica B 255, 1 (1998).

60 B.I. Barker, Y. Lee, L. Polukhina, D.D Osheroff, L.W. Hrubesh, and J.F. Poco, Phys. Rev. Lett. 85, 2148 (2000); B.I. Barker, L. Polukhina, J.F. Poco, L.W. Hrubesh, and D.D Osheroff, J. Low Temp. Phys. 113, 635 (1998).

61 V.V. Dmitriev, I.V. Kosarev, N. Mulders, V.V. Zavjalov, D.E. Zmeev, Physica B 329, 320 (2003).

${ }^{62}$ H. Nakagawa, K. Obara, H. Yano, O. Ishikawa, T. Hata, H. Yokogawa, and M. Yokoyama, J. Low Temp. Phys. 138, 159 (2005).

${ }^{63}$ H. Nakagawa, R. Kado, K. Obara, H. Yano, O. Ishikawa, and T. Hata, Phys. Rev. B 76, 172504 (2007).

64 J.E. Baumgardner and D.D Osheroff, Phys. Rev. Lett. 93, 155301 (2004).

${ }^{65}$ V.V. Dmitriev, L.V. Levitin, N. Mulders, and D.E. Zmeev, JETP Lett. 84, 461 (2006).

66 V.V. Dmitriev, I.V. Kosarev, N. Mulders, V.V. Zavjalov, D.E. Zmeev, Physica B 329, 324 (2003).

67 E. Nazaretski, N. Mulders, and J.M. Parpia, JETP Lett. 79, 383 (2004);J. Low Temp. Phys. 134, 763 (2004).

68 V.V. Dmitriev, V.V. Zavjalov, D.E. Zmeev, I.V. Kosarev, and N. Mulders, JETP Lett. 76, 371 (2002).

69 P. Brussaard, S.N. Fisher, A.M. Guenault, A.J. Hale, N. Mulders, G.R. Pickett, Phys. Rev. Lett. 86, 4580 (2001).
70 G. Baramidze and G. Kharadze, Physica A 284-288, 305 (2000).

${ }^{71}$ H.C. Choi et al., Phys. Rev. Lett. 93, 145302 (2004).

72 H. Choi, Ph.D. thesis, Northwestern University, 2001, (unpublished); H. Choi et al. American Institute of Physics Proceedings, 850, 241 (2006).

73 S.N. Fisher, A.M. Guenault, A.J. Hale, and G.R. Pickett, Phys. Rev. Lett. 91, 105303 (2003).

74 A. Golov, D.A. Geller, and J.M.Parpia, Phys. Rev. Lett.

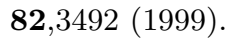

75 H.C. Choi, N. Masuhara, B.H. Moon, P. Bhupathi, M.W. Meisel,Y. Lee Y, N. Mulders, S. Higashitani, M. Miura, and K. Nagai, Phys. Rev. Lett. 98, 225301 (2007).

76 S. Higashitani,M. Miura, M. Yamamoto M, K. Nagai, Phys. Rev. B 71,134508 (2005).

77 D.I. Bradley, S.N. Fisher, A.M. Guenault, R.P. Haley, N. Mulders, S. O'Sullivan, G.R. Pickett, J. Roberts, V. Tsepelin, Phys. Rev. Lett. 98, 075302 (2007).

78 W.P. Halperin and E. Varoquaux, in Helium Three, ed. by W.P. Halperin and L.P. Pitaevskii (Elsevier, Amsterdam 1990).

79 G. E. Volovik, Pis'ma Zh. Éksp. Teor. Fiz. 63, 281 (1996) [JETP Lett. 63, 301 (1996)].

80 Y. Imry and S. Ma, Phys. Rev. Lett., 35, 1399 (1975).

81 I.A. Fomin, J. Low Temp. Phys. 134, 769 (2004).

82 O. Ishikawa, et al., AIP Conf. Proc., 850, 235 (2006).

83 J. Pollanen, J.P. Davis, B. Reddy, K.R. Shirer, H. Choi, and W.P. Halperin, submitted to the proceedings of the 25th International Conference on Low Temperature Physics; ArXive:0806.3927 